\title{
Associative Basis of Landmark Learning and Integration in Vertebrates
}

\author{
Kenneth J. Leising \\ University of Texas Health Science Center at Houston \\ Aaron P. Blaisdell \\ University of California, Los Angeles
}

\begin{abstract}
Early work on spatial navigation evaluated what stimuli (kinesthetic or extra-maze) support small-scale navigation and the nature of the underlying learning (place versus response) process. Contemporary research has focused primarily on how cues interact to determine spatial search. This review covers three general findings from research on landmark-based spatial search in vertebrates. First, pigeons and rats encode simple spatial maps in both open-field and touchscreen environments. Second, a nascent literature shows how simple maps can be integrated into complex maps through higher-order associative processes. The spatial-integration hypothesis provides an associative mechanism for spatial mapping that serves as an alternative to a previously posed configural mechanism. Finally, the evidence for associative cue-competition phenomena in landmark learning is reviewed-focusing on blocking and overshadowing. These findings support a role for associative learning in spatial tasks and provide a powerful explanatory framework for understanding cue integration and competition effects in landmark learning.
\end{abstract}

Keywords: spatial learning, navigation, cognitive map, spatial map, integration, cue competition, overshadowing, blocking, higher-order conditioning.

Animals use many cues to navigate their world. Among these are interoceptive cues, such as proprioceptive and kinesthetic feedback - largely involved in path integrationand external cues such as odors, magnetic fields, and visual landmarks. Although learning is undoubtedly involved in all aspects of spatial behavior, psychologists have focused primarily on how animals learn to use visual landmarks to forage for food or escape from aversive situations. We review the history of spatial learning and evaluate current evidence for the associative basis of spatial learning and navigation, focusing on cue integration and cue competition.

The published history of spatial learning dates back to at

Kenneth J. Leising, Department of Psychology, University of Texas Health Science Center at Houston, Aaron P. Blaisdell, Department of Psychology, University of California, Los Angeles. Support for the production of this paper was provided by $\mathrm{NIH}$ Grant NS059076 (A.P. Blaisdell). Correspondence concerning this manuscript should be addressed to Aaron P. Blaisdell, UCLA Department of Psychology, 1285 Franz Hall, Box 951563, Los Angeles, CA 90095-1563, USA; e-mail: blaisdell@psych. ucla.edu. least the early twentieth century when Small (1901) placed rats in a miniaturized version of the Hampton Court Maze (Figure 1). Small built the maze to study how rats solved 'real life' learning tasks. The maze consisted of a complex series of connected alleys with raised opaque walls to prevent jumping or shortcutting. Rats were required to navigate from a start to a finish location for a food goal. Small made a number of observations regarding the behavior of rats in these experiments, including "the gradually increasing certainty of knowledge", "the clear indication of centrally excited sensations (images) of some kind; memory", and "the almost automatic character of the movements in the later experiments" (Small, 1901, p. 218). Despite his references to memory and cognition, it is the latter comment regarding the automatic nature of learning in the maze that has received the most attention and served as the basis of later studies of navigation in the rat (Carr \& Watson, 1908, Dashiell, 1930; Dennis, 1932; Gingerelli, 1929; Watson, 1907).

\section{Habitual versus Goal-Directed navigation}

Watson believed that like non-spatial learning, spatial learning involved learning stimulus-response (S-R) chains 


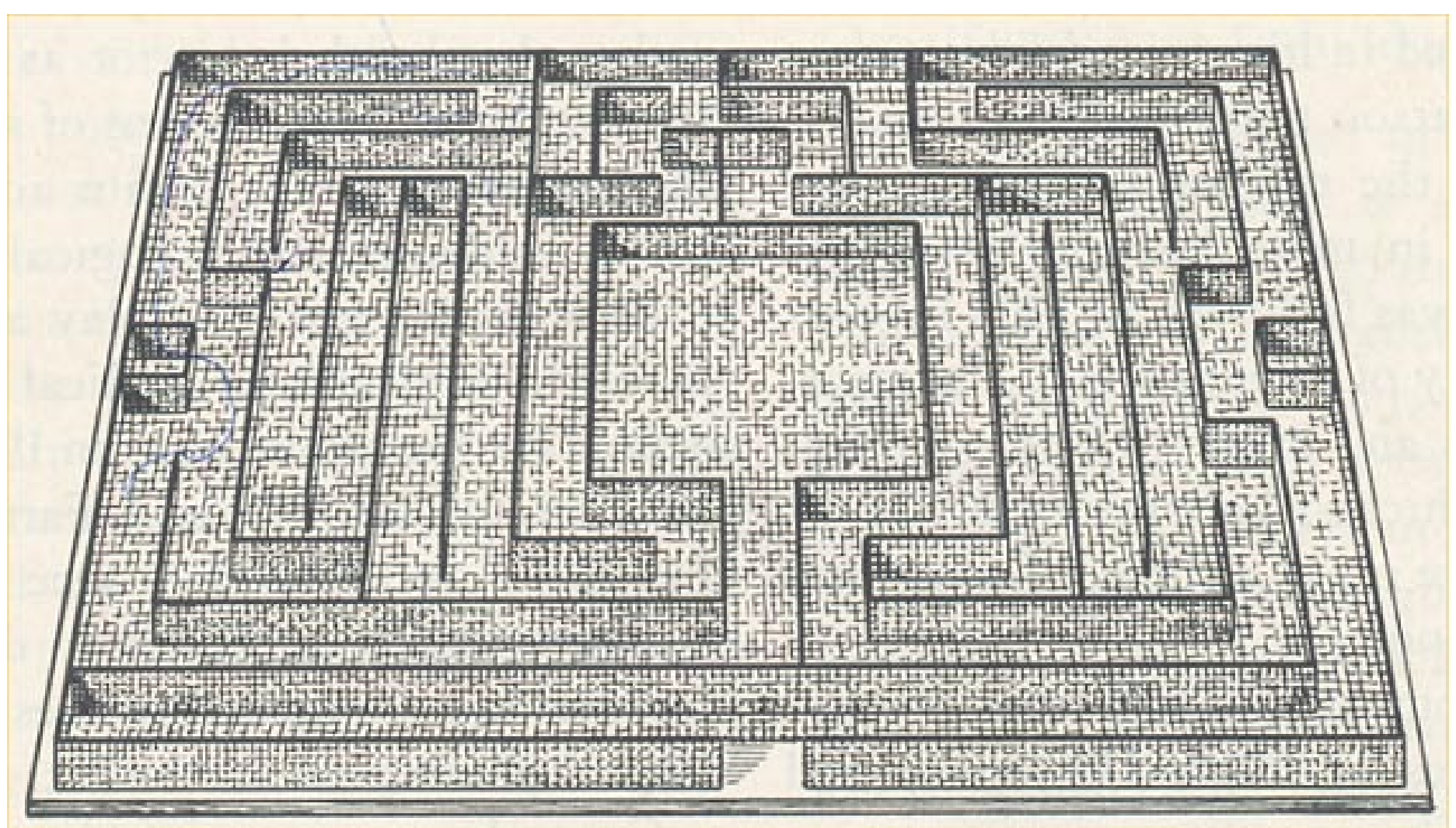

Figure 1. Schematic of Small's Hampton court maze. From "Handbook of psychological research on the rat", by Munn, $N$. L., 1950, Boston: Houghton-Mifflin. Copyright 1950 by N. L. Munn. Reprinted with permission.

as a result of the frequency of pairing a particular stimulus, such as a start box, with a response, such as forward movement. The S-R chain was thought to be initiated by stimuli located at the start box and continued by kinesthetic sensation and muscle movements until the rat reached the reinforcer at the destination. Watson (1907) supported his theory by demonstrating that even rats surgically deprived of sight, hearing, smell, touch, or their combination could navigate the Hampton court maze as well as could normal rats-providing evidence that internal cues such as proprioceptive and kinesthetic feedback were sufficient for navigation.

Carr and Watson (1908) further supported Watson's view while testing rats in a straight-alley maze in which rats were placed at one end and required to run a linear path to the other end to retrieve a food reward. In one experiment, rats first received extensive training in the straight alley maze. After training, the length of the maze was reduced by half and food was placed in front of the new end. Rats ignored the food and ran nose-first into the end of the maze, thereby earning the nickname the 'kerplunk experiment'. The habitual nature of navigation was also supported by other experiments. For example, Stoltz and Lott (1964) found that a large pile of food placed along a well-trained path was repeatedly ignored. Other variations of the kerplunk experiment also found that rats would run nose-first into barriers that had not been present during training (e.g., Dennis, 1932; Gingerelli, 1929).

Although these early experiments conformed to the dominant S-R behaviorist framework of the time, contradictory reports began to emerge showing that navigation behavior could be highly variable (see review by Olton, 1979). One type of apparatus for these studies was the $\mathrm{T}$ or $\mathrm{Y}$ maze (Figure 2). In these mazes, a rat is placed in the start box and faces a left-right choice at the intersection of the arms. Following each choice, the rat is returned to the start box for another trial. In the Y maze rats tend to spontaneously alternate between arms about $80 \%$ of the time (see reviews by Dember \& Fowler, 1958; Douglas, 1966). Similar studies used more complex mazes (e.g., hexagonal) to study patterns of spatial behavior and found that rats rarely return to the previously visited areas before first exploring all remaining areas (Battig, Driscoll, Schlatter, \& Uster, 1976; Uster, Battig, \& Nageli, 1976). Similar results have been obtained in the radial-arm maze (Figure 2), which was developed to study working memory (Olton \& Samuelson, 1976). A radial-arm maze has several arms (typically 8) radiating out from a central platform. If food is placed at the end of each arm, rats quickly learn (e.g., within 40 trials) to alternate arm choice so as to deplete all of the arms with very few revisits to depleted arms. Asymptotic performance often reaches a 

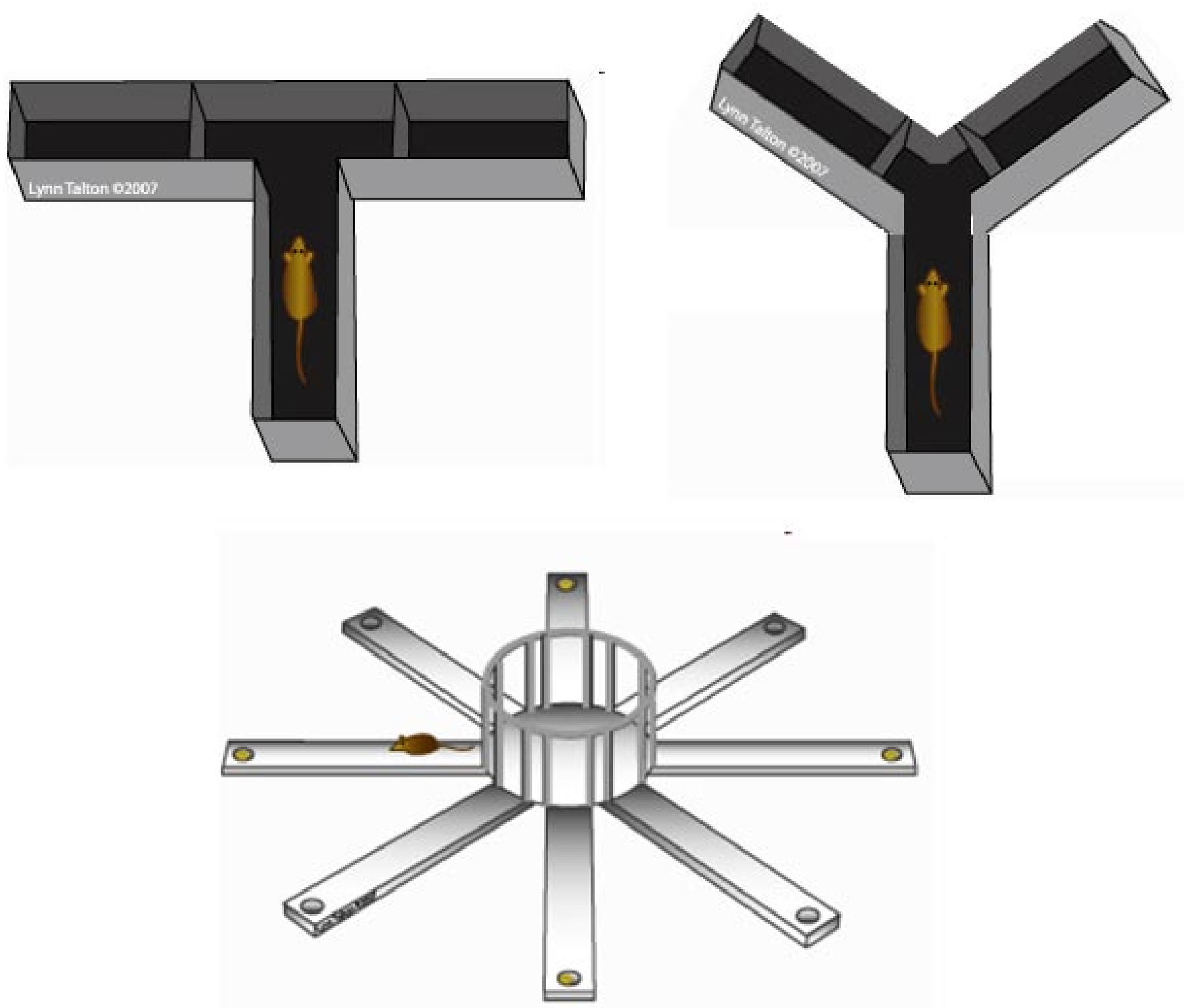

Figure 2. Schematics of the T-maze (top left), Y-maze (top right), and radial maze (bottom). Pictures courtesy of Lynn Talton and the UCLA behavioral testing core (http://btc.bol.ucla.edu/).

mean of 7.90 arms in the first 8 choices (Olton \& Samuelson, 1976). Importantly, from the point of view of navigation, Olton and Samuelson demonstrated that rats' performance was not based on the use of intra-maze cues such as odor trails, subtle variations between arms, or response chains (though rats can, if forced, learn to use intra-maze cues to navigate, see Tremblay \& Cohen, 2005). Rather, rats used extra-maze cues, such as stable visual cues in the room (Olton \& Samuelson, 1976).

Rats not only readily utilize mazes in the laboratory setting; they also spontaneously travel along tunnels in more natural settings. In one remarkable study of rat ecology, Calhoun (1962) introduced rats into a .25 acre enclosed area with internal barriers added to imitate a typical city block. At the center of the enclosure, food and water were made con- tinuously and abundantly available. Additionally, 36 wooden boxes were buried in the ground to serve as burrows. Five pairs of rats were introduced to the area and observations of behavior were made in the two years that followed. During this time, the rats established two main burrows with tunnels radiating out from each burrow toward significant areas of the enclosure, such as food or a nearby buried wooden box. Rats exhibited two types of navigation. The first was to use the shortest or most efficient route between two places, while the second involved longer, windier, and less efficient travel characteristic of investigatory and exploratory behavior. The more cues, goals, or minor obstructions present, the more random the rat's path. Remarkably, rats were very competent in navigating between burrows and food when five inches of snow covered the ground, and constructed subnivian tunnels 
below 15 inches of snow.

Studies using complex mazes, such as the radial-arm maze, hexagonal maze, $\mathrm{T}$ and $\mathrm{Y}$ mazes, or large enclosures - all of which contain one or more choice points - seem to support flexible, goal-directed navigation, while habitual navigation appears more common in the simpler alley maze (see Olton, 1979). Nevertheless, maze complexity is not the only determinant of navigational learning strategy. An important experiment from Tolman's lab revealed that the nature of the rewarded response played a large role in determining the goal-directed versus habitual nature of spatial control in the maze. In one experiment (Tolman, Ritchie, \& Kalish, 1946b), rats were placed on alternate trials at the north or south end of a plus maze. Rats in the response group were always required to make a right turn to find food, whereas, rats in the place group were required to make a different response to find food which was always located in the same place relative to the rest of the room. Performance improved more rapidly in the place than in the response group leading Tolman et al. (1946b) to suggest that, while both types of learning occurred, place learning was a "simpler and more primitive" type of learning than response learning (p. 228). While Tolman's findings have received additional support (e.g., Galanter, 1955; Packard \& McGaugh, 1996), subsequent studies (e.g., Blodgett \& McCutchan, 1948; Scharlock, 1955) as well as a thorough review (Restle, 1957) of the response versus place learning literature identified that rats could find food using either strategy. Moreover, the type of strategy used was largely influenced by the availability of information at the goal and surrounding areas and by the number of training trials. A place strategy (i.e., goal directed) is typically established early in training but gives way to a response strategy (i.e., habitual) with extended training (Hicks, 1964; Packard \& McGaugh, 1996; Ritchie, Aeschliman, \& Pierce, 1950).

\section{Learning about the Environment}

Woodworth's (1938) review of the early literature identified a common tendency for rats to search based on the location of the goal and not on a response tendency. He described this tendency as reflecting a type of learning based on objects and their interrelations in space. Tolman (1948) similarly rejected the response-learning view of navigation and suggested instead that when a rat learns to navigate a maze it encodes an integrated representation of the many stimuli in its surroundings and their relation to the food goal. These representations consisted of the spatial attributes of a context, such as routes, paths, and environmental relationships and determined what responses, if any, were to be released. We will cover Tolman's contribution to the role of spatial representations in the section on cognitive maps below.

The literature from the first-half of the twentieth century clearly suggested that rats could encode and use extra-maze cues to navigate a maze. More recently, Morris (1981) developed a task called the water maze (or Morris maze or Morris pool) to directly study spatial learning in rats (see Figure 3). The Morris water maze is a circular pool with opaque walls and filled with water made opaque (e.g., by adding milk powder). A small platform hidden just below the surface of the opaque water served as a goal location to which the rat could escape from swimming when placed in the water. When a rat is released into the pool at various points, they initially search for an escape route in a seemingly random manner. Once they discover the hidden platform, however, they quickly learn (over just a few trials) to swim directly towards the platform location from any release point in the pool. Learning to use the room cues as landmarks to find the hidden platform is evidenced from a decrease in latency to find the hidden platform over trials, and by measuring the rat's preference for the quadrant containing the hidden platform on probe trials with the platform removed. Various manipulations have established that rats indeed navigate by using distal room cues rather than alternative strategies, such as seeing or smelling the platform or by learning a specific route. For example, rotating the room cues causes the rat to search in a new location within the room, though with the same relative spatial relationship to the landmarks (Morris, 1981; Morris, Hagan, \& Rawlins, 1986). Similar results have been obtained in the radial-maze foraging task (Suzuki, Augerinos, \& Black, 1980).

Pigeons utilize similar mechanisms as rats in navigation tasks. Although Bond, Cook, and Lamb (1981) and Olson

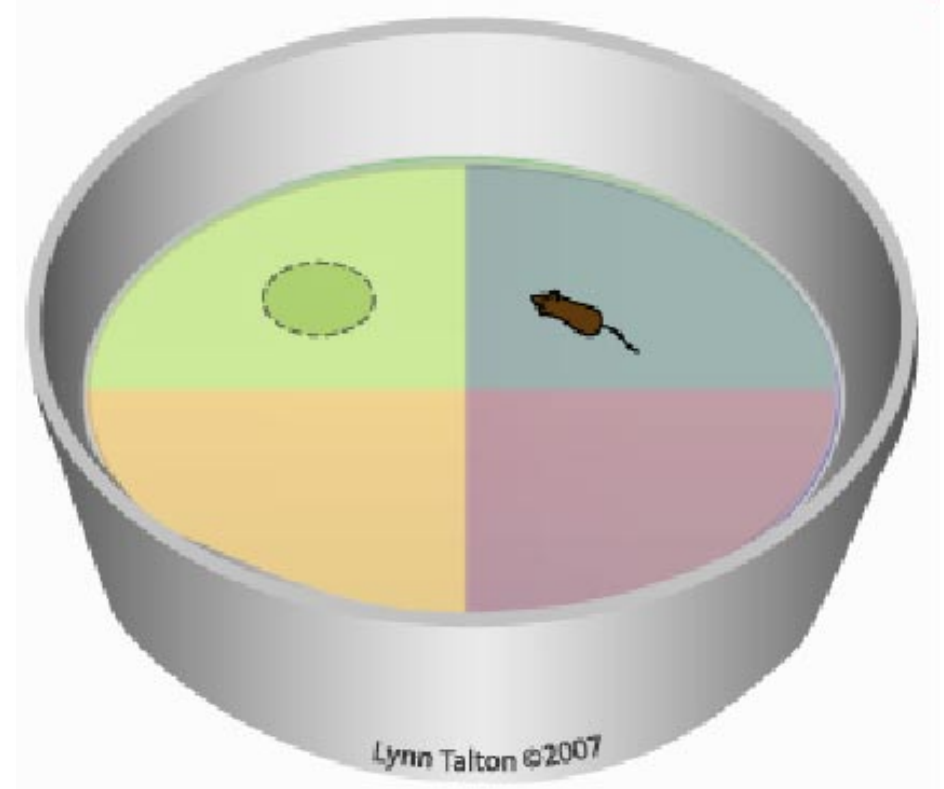

Figure 3. Schematic of the Morris water maze. Courtesy of Lynn Talton and the UCLA behavioral testing core (http:// btc.bol.ucla.edu/). 

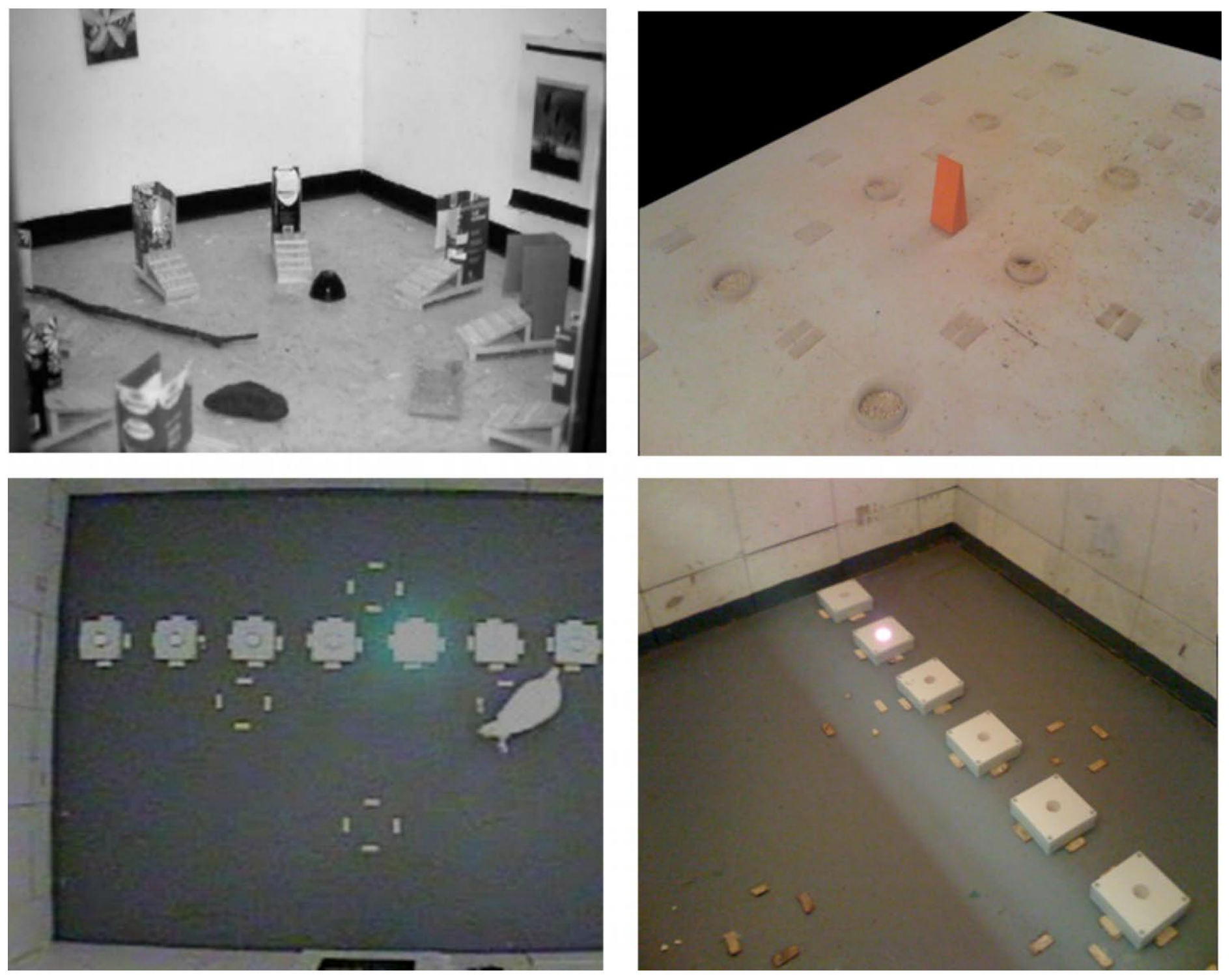

Figure 4. Pictures of open fields used in past spatial learning tasks. Top Left panel: An open field used by Spetch and colleagues with pigeons to approximate the condition of multiple feeders in a radial-arm maze. From "Small-scale spatial cognition in pigeons,” by K. Cheng, M. L. Spetch, D. M. Kelly, \& V. P. Bingman, 2006, Behavioural Processes, 72, p. 116. Copyright 2006 by Elsevier. Reprinted with permission. Top Right panel: An open field used by Stahlman and Blaisdell (2009) to investigate blocking in rats. This open field also closely resembles that used by Blaisdell and Cook (2005) to investigate spatial integration in pigeons. Food can be buried in one of the Tupperware cups and wooden blocks can serve as landmarks. Bottom panels: The general setup of ARENA with modules placed in a linear pattern.

and Maki (1983) both found pigeons to be poorer than rats in a radial-arm maze task, this appears to be due to the fact that traveling through tunnels is not a natural behavior of the pigeon (Roberts \& Van Veldhuizen, 1985). In fact, Spetch and Edwards (1986) removed the tunnels altogether and placing feeders in a circular array in an open field which revealed performance in pigeons that is comparable to that of rats (see Figure 4). Many subsequent studies with pigeons have focused on how they learn to use landmarks while foraging (Cheng, 1988, 1990, \& 1992; Lechelt \& Spetch, 1997; Spetch, 1990, 1995; Spetch, Cheng, \& Mondloch, 1992; Spetch \& Honig, 1988; Spetch \& Wilkie, 1994). We review some of these studies in the following sections.

\section{The Cognitive Map}

Tolman (1948) was one of the first scientists to suggest that animals automatically acquire spatial relationships in simple maze-learning experiments. He coined the term cognitive map to capture the notion that animals acquire a relatively rich representation of their environment during navigation. Tolman's investigations revealed two aspects about how animals learn to navigate. One is the establishment of a place representation and this was the focus of most of Tolman's research. The other is the amount of learning about individ- 
ual stimuli that happens on a given trial. Most associative theories have focused on this latter process (e.g., Hull, 1943; Mackintosh, 1975; N. Y. Miller \& Shettleworth, 2007, 2008: Pearce \& Hall, 1980; Rescorla \& Wagner, 1972) but have paid scant attention to the former.

Tolman's expectancy theory (e.g., Tolman, Ritchie, \& Kalish, 1946a) integrated the effects of reinforcement with learning representations of the environment during training. He proposed that animals learn an expectancy of reinforcement such as food at a particular place, and when stimuli that had been paired with food are presented the animal moves toward the expected location of food. This representation is flexible, such that if the usual route to reward is blocked then the subject will choose an alternate path, or if a new short-cut to reward becomes available then the subject can take advantage of it to move more directly to the food. Tolman (1948) suggested that as the animal navigates it forms an elaborate cognitive map that encodes the spatial relationships among the various features of the room, including the location of reward. The cognitive map is useful for determining what response the animal will emit, given its current position in the map. Tolman also distinguished between two types of cognitive-like maps, a narrow stripmap and a broader comprehensive-map. The strip-map is capable of generating a correct response, but is less flexible when variations are present, such as altered room features or blocked routes. The comprehensive-map represents a wider arc of the environment that flexibly allows responses to be adjusted appropriately. Tolman described the formation of a strip-map rather than a comprehensive-map as induced by a damaged brain, an inadequate array of environmental cues, overtraining, and/or extremely stressful or frustrating conditions - thus foreshadowing much of the contemporary literature on the determinants of goal-directed versus habitual behavior. Tolman's description of the cognitive map was quite vague, but it is clear that what Tolman was trying to capture with these two types of maps was the distinction between goal-directed and habitual, spatially-guided responses.

In their landmark book, O'Keefe and Nadel (1978) put a different twist on the distinction between the response-based strip map and the more flexible comprehensive map. Regarding Tolman's strip maps, these resulted from the operation of a taxon system, which is just another term for the associative-learning system. Thus, strip maps were not really maps at all, but rather were S-R associations. Tolman's comprehensive map, however, was the product of a locale system. O'Keefe and Nadel grounded their theoretical speculations in the emerging neuroscience of the time, showing that habitual responses and responses consistent with a cognitive map were supported by different neural systems. For example, the locale system utilizes place cells in the hippocampus which encode locations in space and enable place learning; while extra-hippocampal areas contribute to the taxon system and result in response learning (O'Keefe \& Nadel). The locale system was said to generate cognitive maps by means of a novelty reduction mechanism (i.e., curiosity), and these maps are constructed in an all-or-none manner (contrary to an elemental associative mechanism). Cognitive maps preserve the Euclidean relationships between features, yet no individual feature is necessary to maintain the relationships among the remaining features (O'Keefe \& Conway, 1978). This type of representation is highly flexible and resistant to interference.

The taxon system, on the other hand, works by attaching valence to a stimulus and encodes stimulus-response commands that lead the subject from one place to another. The taxon system is associative and is subject to associative rules, such as interference between stimuli. In this framework, the taxon system controls behavior when a beacon at the goal is available, when there are few behavioral options, and when there are insufficient cues present for landmark learning to occur, whereas the locale system controls behavior when a rich array of stimuli predict the goal, landmarks are shifted to provide erroneous information, or the route from a landmark to a goal is blocked. The designation of a locale and taxon system for navigation has inspired decades of research. While some of O'Keefe and Nadel's (1978) formulations have survived rigorous evaluation, many of them have not (Chamizo, 2002; Sherry and Healy, 1998), and thus there have been attempts to reformulate the mechanisms of spatial learning.

The most recent incarnation of a two-system theory of spatial cognition comes from Burgess $(2006 ; 2008)$. Burgess argues that learning about landmarks and beacons is supported by egocentric representations (i.e., taxon) while incidental learning of environmental boundaries is supported by allocentric representations (i.e., locale). Allocentric spatial coding is the encoding of the Euclidian spatial relations of distance and direction among objects in external space. Egocentric spatial coding is the encoding of the spatial relationship between the subject and external cues such as landmarks or goals. Burgess (2008) eloquently describes ample evidence to support an egocentric-allocentric division based on the encoding of sensory and spatial information by dorsal and ventral processing streams. The major departure here is that the allocentric representation suggested by Burgess is limited to environmental boundaries, whereas, O'Keefe and Nadel (1978) proposed that all features in the environment would be encoded into a cognitive map. Consistent with cognitive map theory, the dual representations occur in parallel, and which representation operates on behavior is dependent on the task demands as well as the timescale. In describing how allocentric representations are constructed, Burgess hypothesized that the construction and behavioral 
manifestation of an allocentric representation necessarily relies both on cells coding for absolute location (i.e., place and grid cells) and the translation of spatial information to and from egocentric representations (e.g., viewpoint dependent and path integration). Thus, at some level these representations interact. Although Burgess dismisses competition between representations, there is both the mechanism for, and behavioral evidence to support, such interaction (facilitation and competition).

\section{A General Mechanism of Spatial Learning}

Discussions about spatial learning mechanisms have focused on the role of geometric and featural cues (Cheng, 1986), directional and positional cues (Jacobs \& Schenk, 2003), geocentric and egocentric vectors (Gallistel \& Cramer, 1996), and topological and metric information (Poucet, 1993). Though these distinctions have proven valuable for driving research on spatial cognition, they are not without dispute (Chamizo, 2002, 2003; Cheng, 2008). In fact, it would be more parsimonious to try to account for all allocentric spatial learning-encoding the Euclidian spatial relations of distance and direction among objects in external space - with a single general associative mechanism rather than posit multiple specialized and independently functioning mechanisms. There is growing evidence to support a single mechanistic account of allocentric spatial learning (see attempt to do so by N. Y. Miller \& Shettleworth, 2007,

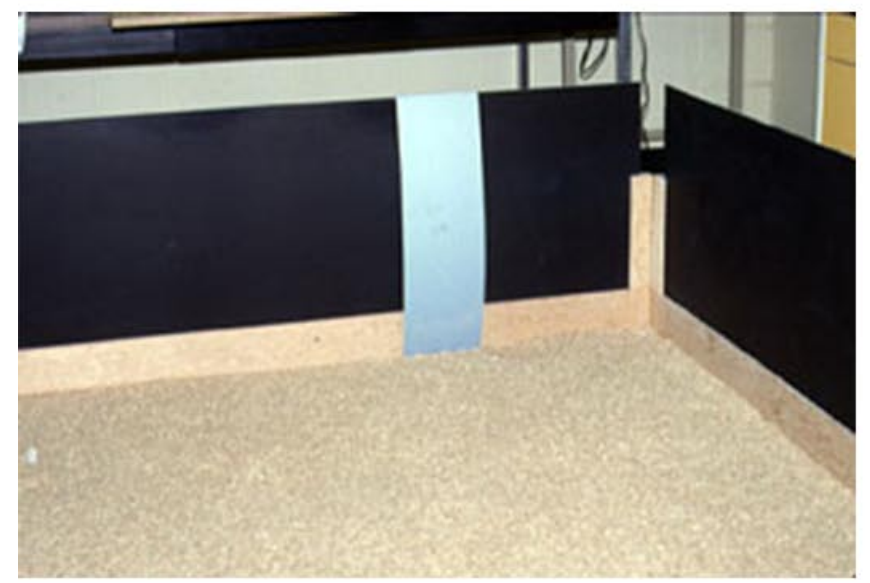

2008). Furthermore, Burgess (2008) indirectly provides the theoretical and physiological link for the interaction between egocentric representations of landmarks and allocentric representations of space. He suggests the necessary translation of egocentric into allocentric and allocentric into egocentric. This interaction provides an opportunity for competition both at the time an allocentric representation is translated into behavior, as well as when lower level features of landmark representations - such as viewpoint information - are translated into allocentric maps. We are attempting in this review to explore the role of associative processes in constructing and using allocentric spatial relationships specifically. Associative processes may or may not also play a role in egocentric spatial relationships - those between the subject and points in external space-but this review does not address this issue. The following sections review the evidence for such an account from studies on landmark learning, landmark integration, and landmark competition. In the final section we discuss the types of associative models that best account for landmark learning.

\section{Landmark learning}

Marcia Spetch and Ken Cheng have extensively explored how pigeons learn to use landmarks to find hidden goals. This work has been carried out in both the open field and in touchscreen-equipped operant chambers. In the basic landmark-based search task, the pigeon is reinforced for

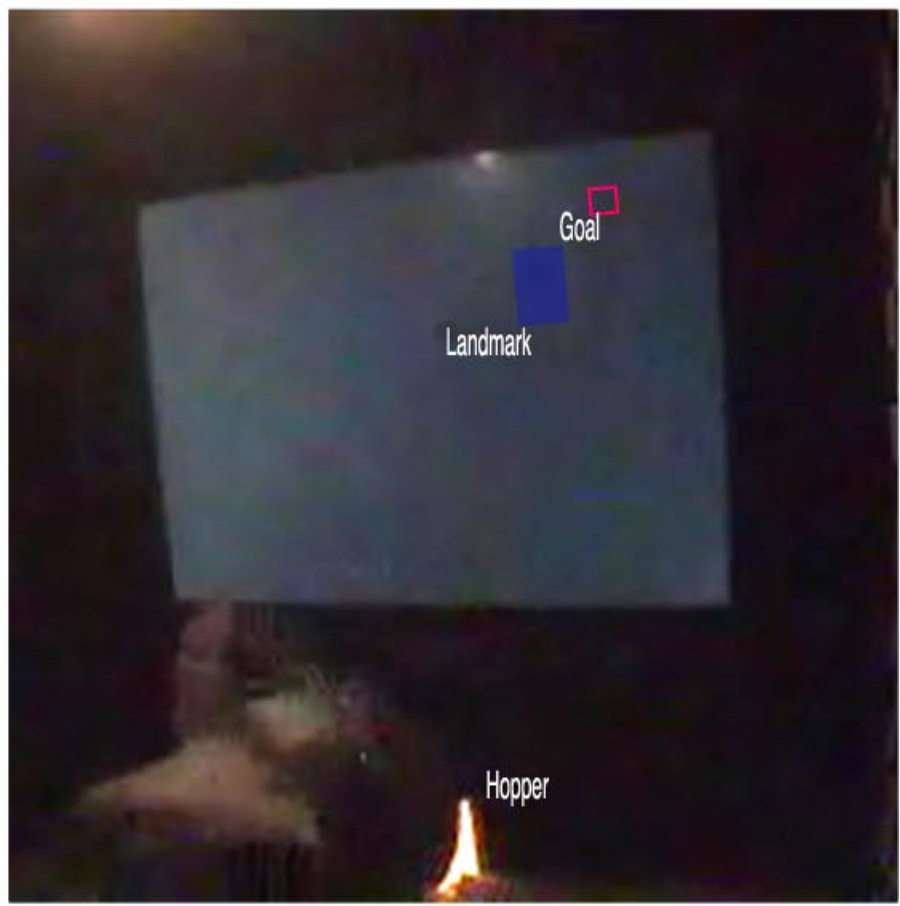

Figure 5. Left panel: Picture of an open-field apparatus from Cheng and Spetch (2001). From "Landmark-based spatial memory in pigeons,” by K. Cheng \& M. L. Spetch, 2001, In Avian visual cognition [On-line]. Available: www.pigeon.psy. tufts.edu/avc/cheng/, R. G. Cook (Ed.). Copyright 2001 by K. Cheng \& M. L. Spetch. Reprinted with permission. Right panel: screen shot of a touchscreen procedure from our laboratory. 
locating a food goal buried under a substrate such as sand or wood chips on the floor of an open field or for pecking at an unmarked location on a touchscreen for which they are reinforced from a food hopper (Figure 5). Cheng (1988; 1989) reported that how pigeons learn to use landmarks to find a hidden goal on the floor of an open field conformed to predictions of the vector sum model of landmark-based navigation originally formulated by Collett, Cartwright, and Smith (1986). According to the vector sum model, pigeons (or any animal) that learn to use a visual landmark to find a hidden goal encode the spatial vector between landmark and the goal. A vector is a metric specifying both distance and direction between two points in space-and can be used to encode the spatial relationship between any two objects. The direction is coded in reference to a larger framework of orientation, such as the sides of the touchscreen monitor or the walls of the room containing the open field. A vector is easily conceptualized as an allocentric spatial map between the two objects, for example, the distance and direction between Object A and Object B. Object A may be a junk object located in the open field and Object B may be a food goal buried under sand on the floor of the open field. Alternatively, Object A may be a colored shape presented on the surface of the touchscreen and Object B may be a spatial location on the monitor at a fixed distance and direction relative to Object A.

When multiple spatial cues are available during learning, the vector sum model provides rules by which the various landmarks are weighted by the subject. For example, landmarks proximal to the goal are weighted more heavily than are landmarks further from the goal. Evidence for this comes from manipulations such as shifting the closer or more distal landmark and observing the effect on the location of search on a non-reinforced probe test (Cheng, 1989; Spetch et al., 1992; Spetch \& Mondloch, 1993). When the location of a proximal landmark is shifted, this causes a greater shift in the peak search location; whereas when the location of a distal landmark is shifted, smaller shifts in peak search location are observed. Further evidence that proximal landmarks are weighted more heavily than distal landmarks comes from studies of a spatial analog of the overshadowing effect (Lechelt \& Spetch, 1997; Spetch, 1995; see below). Another concern of the vector sum model is in how subjects measure perpendicular distance from extended surfaces, such as a wall or border. Extended surfaces play a substantial role and affect computations by the vector sum model (see Cheng, Spetch, Kelly, \& Bingman, 2006, for a discussion of these issues). There is now a wealth of evidence supporting the vector sum model of landmark-based spatial search coming from a variety of species, including pigeons, humans, rodents, and insects (Cheng, 1994; Cheng \& Spetch, 1995, 1998; Kamil \& Cheng, 2001; Spetch, Cheng, \& MacDon- ald, 1996; Spetch, Cheng, MacDonald, Linkenhoker, Kelly, \& Doekson, 1997; Spetch et al., 1992; Spetch \& Mondloch, 1993).

\section{Integration of Spatial Maps}

If animals can acquire simple spatial maps-such as a vector between a landmark and a goal-through associative processes, then the associative processes underlying higherorder conditioning may serve to integrate multiple spatial maps between landmarks and goals. Higher-order conditioning involves establishing a conditioned response to a CS that has a second-order (and therefore indirect) relationship to the US. This response is established through pairings between the second-order CS2 and the first-order CS1 (i.e., $\mathrm{CS} 2 \rightarrow \mathrm{CS} 1$ ) and through pairings of CS1 with the US (i.e., CS1 $\rightarrow$ US). This can be accomplished either in a second-order conditioning procedure in which CS $1 \rightarrow$ US pairings occur prior to $\mathrm{CS} 2 \rightarrow \mathrm{CS} 1$ pairings, or in a sensory preconditioning procedure in which CS2 $\rightarrow$ CS1 pairings occur prior to $\mathrm{CS} 1 \rightarrow$ US pairings.

According to this spatial-integration hypothesis, more complex representations can be built by linking together simpler representations that share common elements or events. The spatial-integration hypothesis extends on prior work by Ralph Miller and colleagues showing that temporal integration occurs during higher-order conditioning procedures (Arcediano, Escobar, \& Miller, 2003; Leising, Sawa, \& Blaisdell, 2007; Savastano \& Miller, 1998). For example, Leising et al. used an appetitive sensory preconditioning procedure with rats consisting of a 60 -s CS2 paired with a 10-s CS1 in Phase 1, followed by the 10-s CS1 simultaneously paired with sucrose US in Phase 2, followed by nonreinforced probe tests on CS2 (see Figure 6). During Phase 1, CS1 was presented during either the early or later portions of CS2 for rats in Group Early and Late, respectively. If rats acquired a CS2 $\rightarrow$ CS1 temporal map during Phase 1, and then linked this map with the CS1 $\rightarrow$ US map acquired in Phase 2, then rats in Group Early should expect food during the early portion of the CS2 test trials, while rats in Group Late should expect food during the later portion of the CS2 test trials. These predictions were confirmed by rats in Group Early showing greater anticipatory nose poking for food during the early portions of CS2 while rats in Group Late made more anticipatory nose pokes later during CS2 at test. These results demonstrate that an associative integration process can link together separate temporal maps into a higher-order, unified map.

Higher-order associative processes have been found to play a similar role in spatial integration (Blaisdell \& Cook, 2005; Sawa, Leising, \& Blaisdell, 2005). Blaisdell and Cook (2005) used an instrumental analog to the Pavlovian 

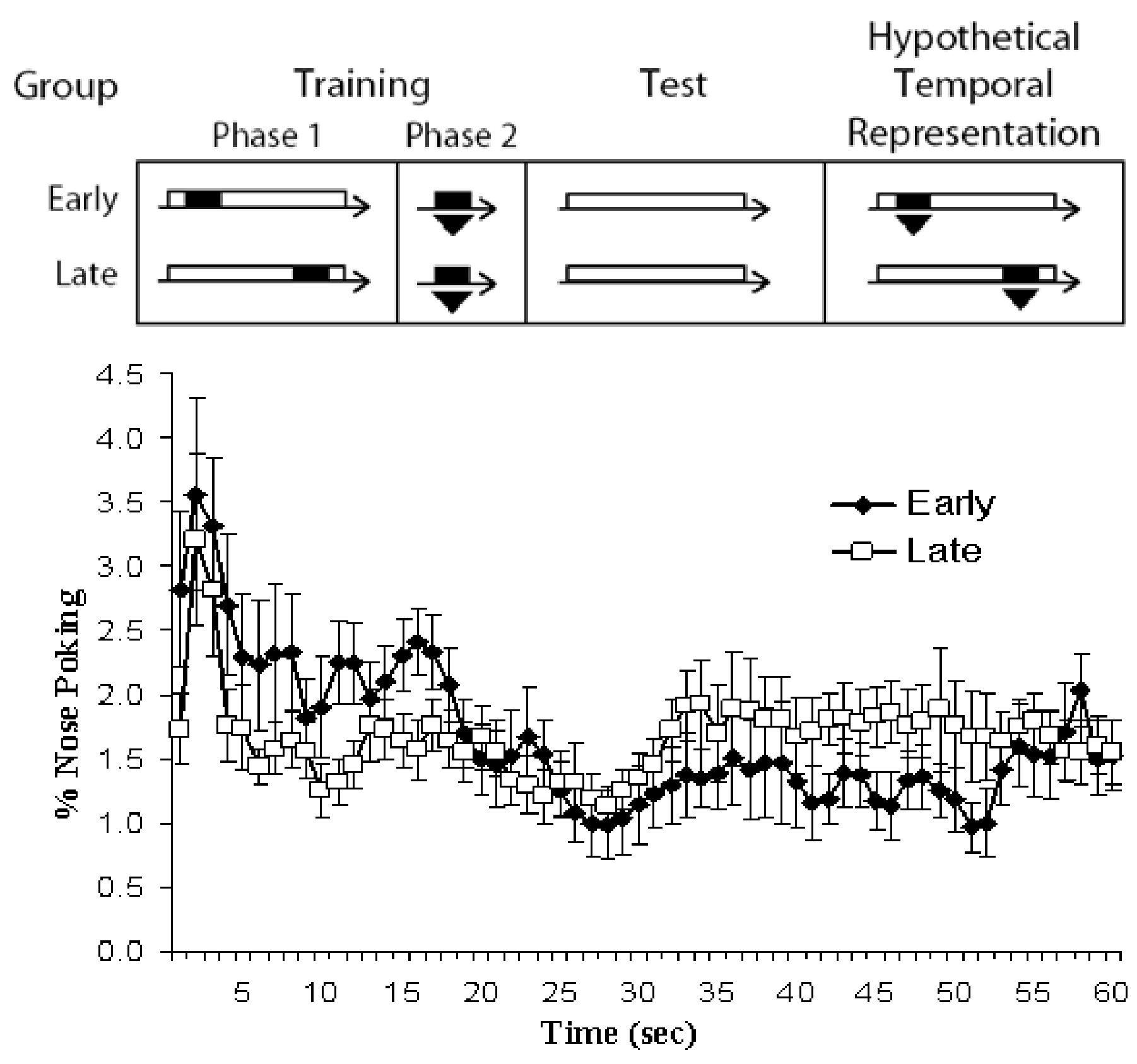

Figure 6. Top panel: Design and hypothetical temporal representations of Experiment 1 of Leising et al. (2007). A 60-s stimulus served as the light and a 10-s stimulus served as the tone. The US was access to a .05 cc cup of sucrose solution. Bottom panel: mean normalized magazine entries during the light at test (in 1-s bins) for Groups Early (filled symbols) and Late (open symbols). From "Temporal integration in Pavlovian appetitive conditioning in rats," by K. J. Leising, K. Sawa, \& A. P. Blaisdell, Learning \& Behavior, 35, p. 13 \& 14. Copyright 2007 Psychonomic Society, Inc. Adapted with permission.

sensory-preconditioning procedure to study the integration of spatial maps in an open-field search task in pigeons (see Figure 7, left panel). Pigeons were initially trained to find grain hidden in one of 16 gravel-filled cups (see Figure 4 for a similar apparatus). On each trial, one of the 16 cups was randomly designated the goal where food was then hidden. After pigeons had learned to search for food, they received sensory preconditioning treatment. On sensory-preconditioning trials, pigeons were presented with two visual landmarks (LM1 and LM2; LM1 was a T-shaped, LM 2 was an L-shaped piece of wood) placed on the floor of the open field. The landmarks always had a stable spatial relationship to each other (LM2 $\rightarrow$ LM1) but were placed at various locations in the open field across trials. Food was buried in the cup located between the landmarks to ensure that pigeons attended to them. Subsequently, pigeons learned to find the hidden goal at a new location relative to LM1 (the T) which was placed at a fixed distance and direction from a hidden 
food goal (LM1 $\rightarrow$ Goal). In both phases, the location of the goal within the open field varied randomly across trials, so the only way the pigeons could find it at above chance levels was by attending to the $\mathrm{LM} 1 \rightarrow \mathrm{Goal} \leftarrow \mathrm{LM} 2$ spatial relationship in Phase 1 , and to the $\mathrm{LM} 1 \rightarrow$ Goal spatial relationship in Phase 2. Finally, pigeons received nonreinforced probe tests with LM2 (the L) alone. If they had encoded the LM2 $\rightarrow$ LM1 and LM1 $\rightarrow$ Goal spatial maps, they could have integrated the maps to form a LM2 $\rightarrow$ LM $1 \rightarrow$ Goal spatial map, from which they could compute a LM $2 \rightarrow$ Goal relationship. Pigeons did show evidence for making such a computation as evidenced by the greater responding at the goal location based on integration of the Phase 1 and Phase 2 spatial maps than at any other location (Figure 7, right panel).
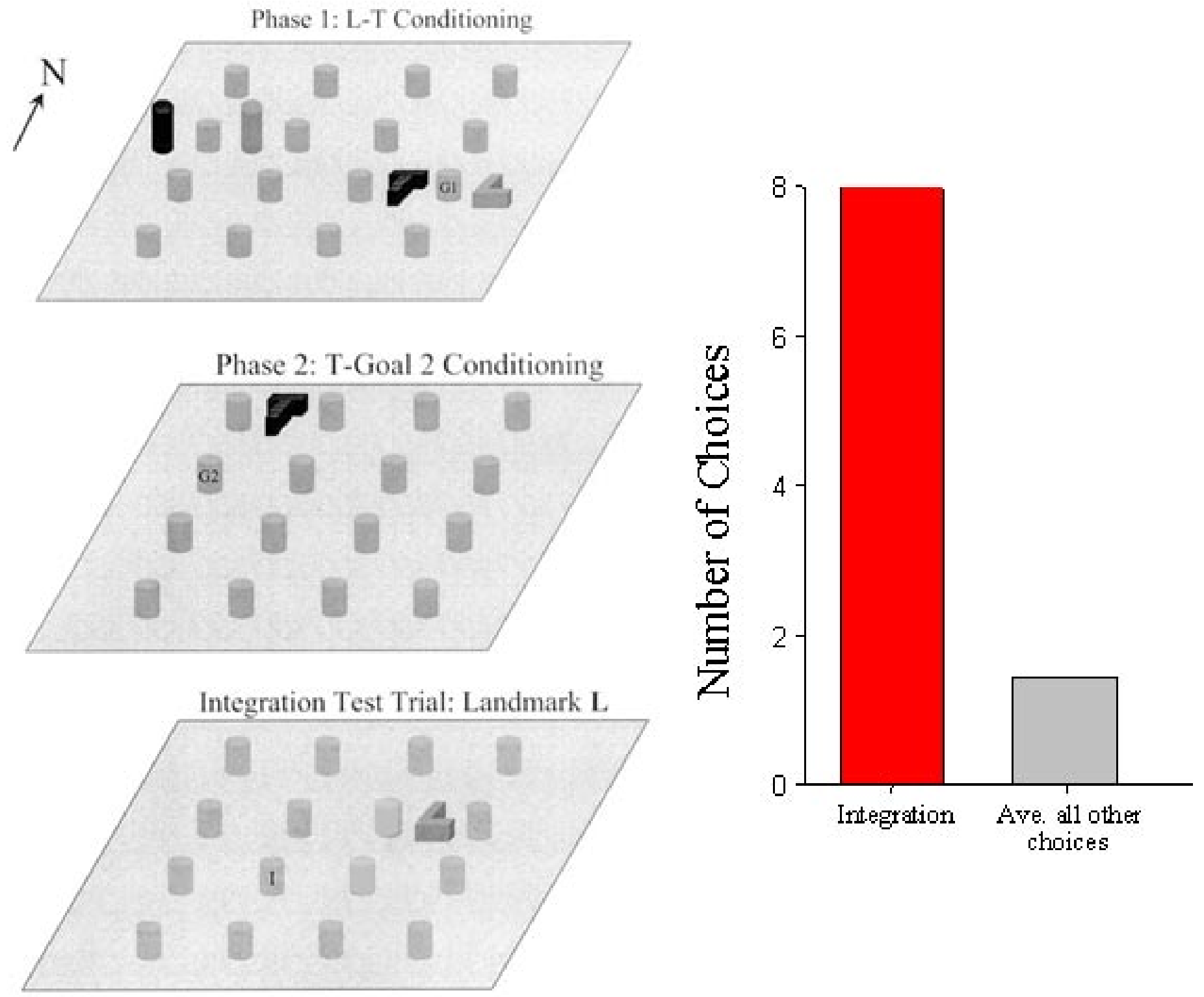

Figure 7. Left panel: Diagram of the experimental arena used by Blaisdell and Cook (2005), showing the arrangement of the $4 X 4$ grid of gravel-filled cups, the hidden food $(G)$, and the landmarks ( $T, L$, and two foils). The top panel shows the spatial arrangement of the consistent landmarks (T and L), goal 1 (G1), and inconsistent landmarks (cylindrical foils) during phase 1. The middle panel shows the spatial arrangement of LMT to goal $2(G 2)$ during phase 2. The bottom panel shows the spatial arrangement of LM L and the potential locations of search during the integration test. Letters on bottom panel: $I=$ predicted cup for choices guided by the $L \rightarrow T \rightarrow$ Goal 2 hierarchical map, $A=$ predicted cup for choices guided by the phase $1 \mathrm{~L} \rightarrow$ Goal 1 vector, and $G=$ predicted cup for choices guided by a generalization to $L$ of the $T \rightarrow$ Goal 2 vector. Right panel: Mean choices at the Integration location and at all other locations on test trials with LM L. From "Integration of spatial maps in pigeons,” by A. P. Blaisdell \& R. G. Cook, 2005, Animal Cognition, 8, p. 9. Copyright 2004 by SpringerVerlag. Adapted with permission. 
The results of the experiment by Blaisdell and Cook (2005) have been criticized, in part, due to the inclusion of a food goal during Phase 1 sensory-preconditioning trials $($ LM1 $\rightarrow$ Goal $\leftarrow$ LM2; Sturz, Bodily, \& Katz, 2006). Sturz et al. replicated the design of their experiment using a virtual 3D computer gaming environment with human subjects but found no evidence for spatial integration. Instead, performance on the first test trial appeared to generalize from the LM1 $\rightarrow$ Goal map established in Phase 2. Over additional test trials, subjects' searches progressively shifted away from the test landmark in all directions. This performance reflects a failure of the human subjects to integrate the separate phases of training into a coherent spatial map.

Sawa et al. (2005) have replicated the design of Blaisdell and Cook's (2005) experiment in pigeons using a touchscreen-equipped operant box. One important difference was the use of a true sensory-preconditioning design in which a)

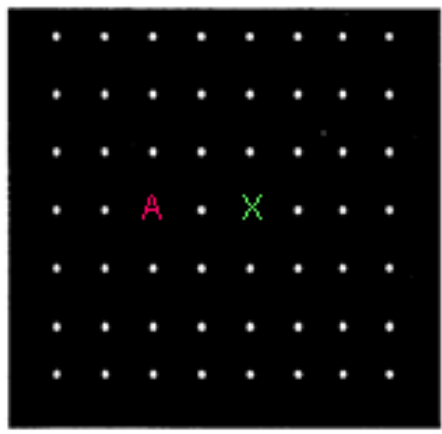

b)

c)

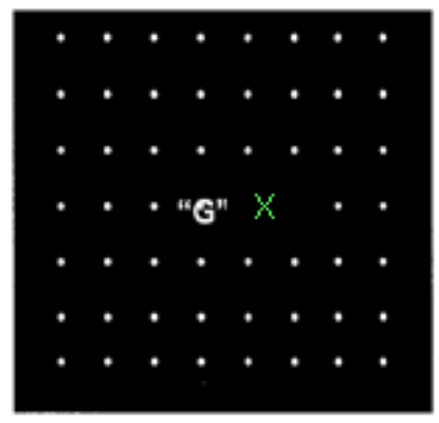

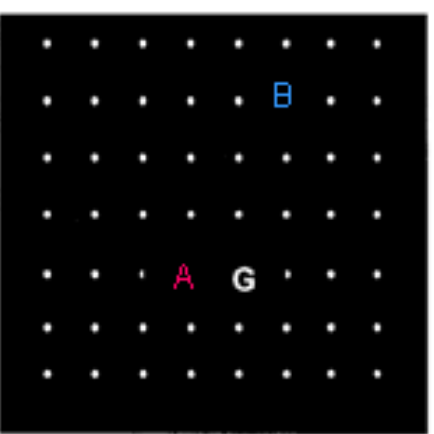

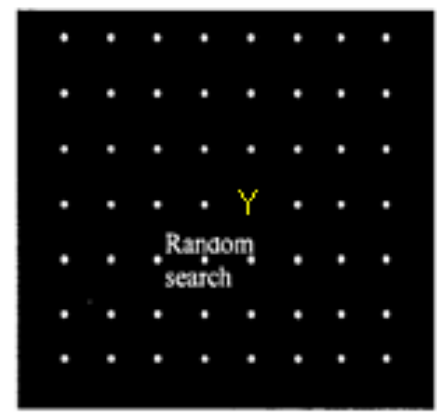

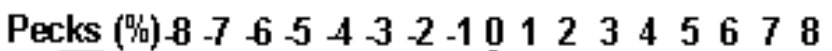
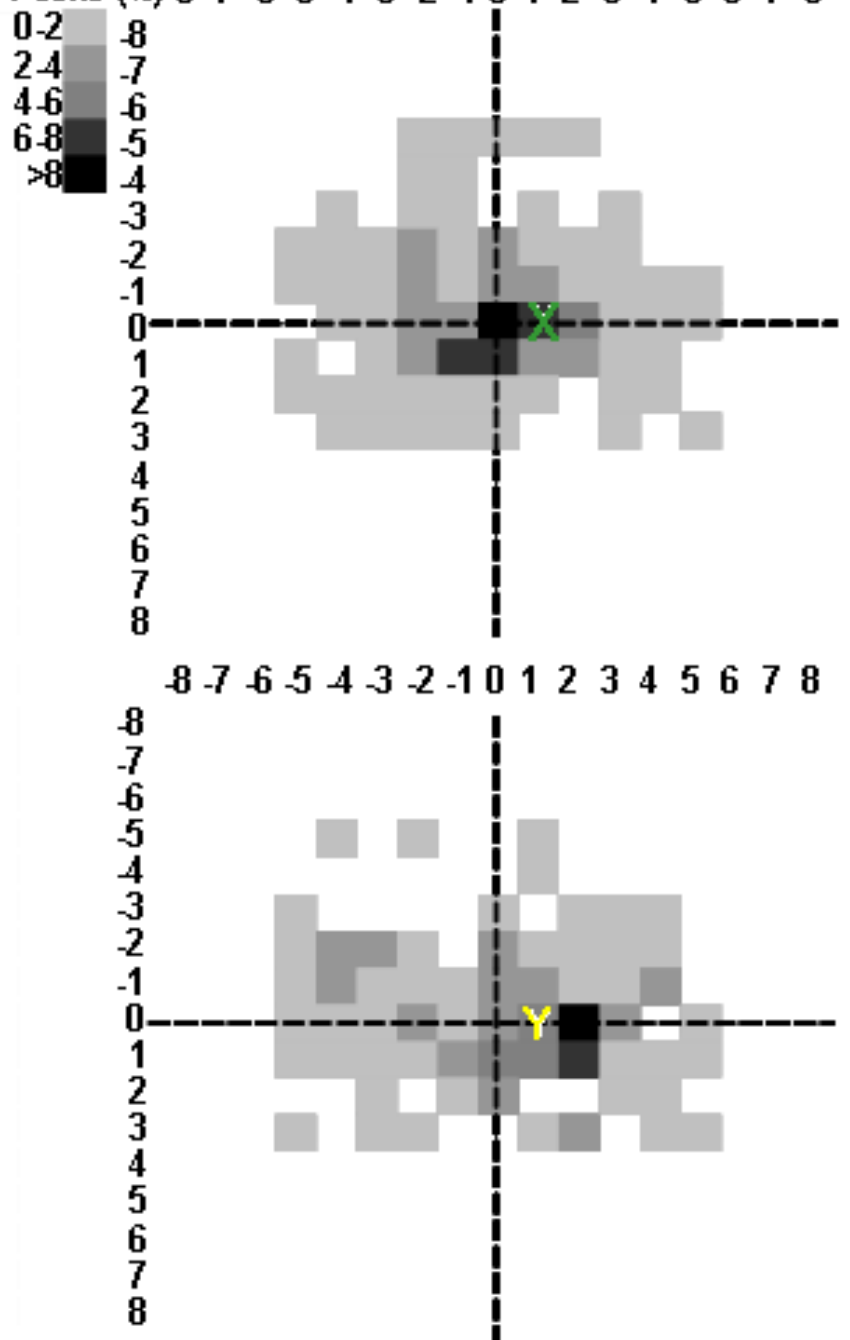

Figure 8. Left panel depicts diagrams of the training and testing landmark (LM) configuration on the touchscreen used by Sawa, Leising, and Blaisdell (2005). From "Sensory preconditioning in spatial learning using a touch screen task in pigeons,” by K. Sawa, K. J. Leising, \& A. P. Blaisdell, 2005, Journal of Experimental Psychology: Animal Behavior Processes, 31, p. 371. Copyright 2005 by the American Psychological Association. Adapted with permission. The circles denote the layout of the response grid. A: spatial relationship between LMs A and X and LMs B and Y during Phase 1 of sensory preconditioning. B: relationship between the consistent LM A to the goal during Phase 2; LM B was also present on Phase 2 trials, but its spatial relationship to the goal was randomly determined. C: predicted peak location of search on test trials with LM X and LM Y; "G" indicates the expected goal location based on the integration of the LM A-Goal and LM X-LM A spatial vectors. "Random search" indicates the expected pattern of search on LM Y trials. Right panel shows density of peck locations on nonreinforced test trials with LMX (top panel) and LM Y (bottom panel). Location $(0,0)$ on the axes indicate the training goal location. 
no food reinforcement was provided during Phase-1 LM2LM1 pairings. The design of each phase is depicted in the left panels of Figure 8. In Phase 1, pigeons received compound presentations of LM A and LM X on some trials and of LM B and LM Y on other trials. LMs A and B served as first-order landmarks (i.e., LM1) while LMs X and Y served as second-order landmarks (i.e., LM2). Each element of the compound maintained a stable within-compound spatial relationship (as depicted in Figure 8) but the screen location of the compounds varied across trials. No food goal was present on these trials. Each Phase 1 trial was presented for $30 \mathrm{~s}$ and ended without reinforcement. In Phase 2, pigeons learned to find the hidden goal in the presence of LM A and LM B. The hidden goal $(\mathrm{G})$ always bore the same spatial relationship to LM A (as depicted in Figure 8) but its spatial location relative to LM B was randomly determined. (The food was delivered from a hopper when the pigeon successfully pecked at the goal location.) Thus, pigeons could only learn to efficiently find the hidden goal by attending to its spatial relationship to LM A, but not to LM B. At test, pigeons received non-reinforced tests of LM X and LM Y on separate trials. Each test trial consisted of a 30-s presentation of either LM X or LM $\mathrm{Y}$ followed by the intertrial interval and no reinforcement. If pigeons are able to integrate the X-A spatial relationship in Phase 1 and the A-Goal relationship in Phase 2, then they may integrate these maps to form a $\mathrm{X} \rightarrow \mathrm{A} \rightarrow$ Goal spatial map. This map would allow the pigeons to compute the $X \rightarrow$ Goal spatial relationship which should guide the peak location of search on test trials. Because the X-A and A-Goal spatial relationships were stable during training, integration of these maps should lead pigeons to search to the immediate left (see Figure 8) of LM X at test. Although the Y-B spatial relationship during Phase 1 was stable, the B-Goal spatial relationship during Phase 2 was not. Thus, even if pigeons integrated the Y-B and B-goal associations, this integrated association should not allow the pigeons to compute a particular Y-Goal spatial map and we therefore predicted searches to be randomly distributed around LM Y.

The spatial distribution of response frequency is shown in the right panel of Figure 8. Consistent with the predictions of spatial integration, the frequency of searches during tests of LM X were clustered around the response location to the left of LM X. This indicates that pigeons had encoded the $\mathrm{X}-\mathrm{A}$ and A-Goal spatial maps and integrated them to allow computation of an X-Goal map. Interestingly, searches during tests of LM Y were not randomly distributed around LM Y. Rather, they were concentrated at a location to the right of LM Y. Sawa et al. (2005) suggested that without any reliable spatial relationship between LM Y and the goal, pigeons may have generalized responding from the well-trained LM A from Phase 2. That is, with no spatial information available, a well-trained response was elicited. Importantly, due to the absence of any reinforcement during Phase 1 of sensory preconditioning, this experiment avoids the type of search strategy Sturz et al (2006) found their human subjects to use. Rather than using the test landmark as a beacon from which to search around, the frequency of searches in the presence of LM X were concentrated to one side of the landmark with far fewer searches on the other side (or above or below LM X). Molet and Miller (2008) recently presented evidence for successful spatial integration in humans using a similar task to that of Sawa et al.

Although the simplest form of integration involves the combining of three elements presented two at a time, as in the pigeon studies described above, spatial integration often involves the combination of compounds made up of many elements - such as a configuration of landmarks. Chamizo, Rodrigo, and Mackintosh (2006) found evidence in rats for spatial integration among configurations of landmarks suspended above the edge of a Morris pool. Importantly, integration occurred only when the configurations shared a common element. Rats in the integration group received alternating trials of a three-landmark configuration (A, B, $\mathrm{C}$, and $\mathrm{C}, \mathrm{D}, \mathrm{E}$ ) with $\mathrm{C}$ as the common element in both configurations (apparatus schematic depicted in Figure 9). Rats in the control group were given similar sets of configurations (A, B, C and D, E, F) but with no common element between

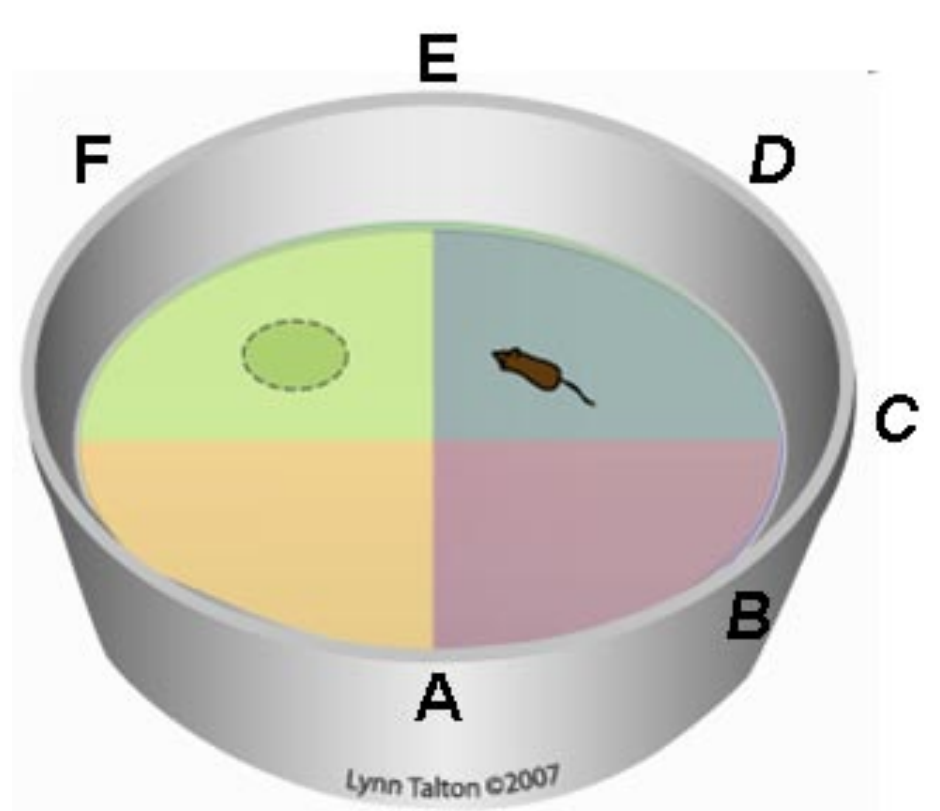

Figure 9. Schematic representation of the pool used by Chamizo, Rodrigo, and Mackintosh (2006). Letters denote the landmarks surrounding the pool. The dark green circle in the pool indicates the location of the hidden platform during training. Copyright by Lynn Talton and the UCLA behavioral testing core (http://btc.bol.ucla.edu/). Adapted with permission. 
them (Experiment 2). Rats were tested on novel configurations of two and three landmarks without the common element, LM C. If integration of spatial information requires a common element as suggested by the results of Blaisdell and Cook (2005) and Sawa et al. (2005) then the integration group should show superior spatial performance relative to the control group on novel configurations without the common element. Indeed, the integration group showed better spatial control by novel two and three landmark configurations than the control group. These findings further support the critical role of a common element to link and integrate spatial maps acquired on independent conditioning trials.

We would be remiss not to cover reports of failures to find integration of cognitive maps (also see discussion by Mackintosh, 2002 and Shettleworth, 1998). Although there are many demonstrations of cognitive mapping that have not held up under careful scrutiny (see Bennett, 1996 for a good review), we know of only one reported failure to find cognitive mapping in nonhuman vertebrates. Benhamou (1996) reported a series of experiments in which rats were placed in a Morris water maze from which they could escape by locating a hidden platform. Unlike the apparatus used by Chamizo et al. (2006) which provided the rats with a full $360^{\circ}$ view of the distal room cues, the apparatus in Benhamou's experiment included an opaque cylinder inserted in the pool which separated a small peripheral area around the edge from the inner area of the pool. The cylinder had a single opening allowing access between the interior and exterior of the cylinder. During training rats were placed outside the cylinder and required to swim from the peripheral area to the inner area to find the hidden platform. Once inside, the opening in the cylinder provided only a $90^{\circ}$ view of the distal room cues. This contrasts markedly from the apparatus used by Chamizo et al. (2006) which provided the rats with a full $360^{\circ}$ view of the distal room cues. Tests were conducted with the cylinder rotated so that the only available distal cues observable at the platform were those that had not been observable from the platform during training. On these novel transfer tests rats were unable to locate the hidden platform accurately and efficiently. This was taken as evidence that rats had not integrated all of the room cues to form a cognitive map. A problem with this procedure is that the cylinder provides a very salient local cue that may receive greater weighting and thus detract from (i.e., overshadow) use of distal cues (Chamizo, Manteiga, Rodrigo, \& Mackintosh, 2006; Spetch, 1995). Even when the situation was such that use of distal cues was encouraged (Experiment 3), performance was still poor relative to baseline, and poorer than that shown in previous studies where a $360^{\circ}$ view of distal cues was available. Thus, while creative, this procedure does not provide a stringent test of cognitive mapping.

A more recent replication of a similar procedure with
Clark's nutcrackers found that some subjects were able to find a hidden food goal using a novel view of distal cues (Gibson \& Kamil, 2001). This experiment is subject to the same criticisms as those by Benhamou (2006) described above. Moreover, the authors acknowledged that the successful birds could have located the hidden goal by an alternative strategy that uses stored landmark-goal vectors and a compass bearing provided by polarizing room cues.

The procedures used by Blaisdell and colleagues in pigeons and by Chamizo, Rodrigo, and Mackintosh (2006) in rats provide access to only a subset of cues during each phase of training and thus the only solution to accurately locate the hidden goal necessitates the integration of the separate sets of cues into a common representation. No other non-mapping spatial mechanism will do. Thus, these studies provide the strongest support of spatial integration - a key, unique feature of cognitive mapping. But, in contrast to O'Keefe and Nadel (1978), these studies suggest that associative mechanisms of higher-order conditioning can serve to integrate spatial information in piecemeal fashion into a cognitive map. These findings provide support for the spatial-integration hypothesis. According to this hypothesis, complex representations can be built by linking together simpler representations that share common elements or events. The simplest conceivable spatial association encodes the spatial relationship between two events, such as two landmarks, or a landmark and a food goal. A spatial representation containing three events can be built in one of two ways. On the one hand, all three events could be presented simultaneously, in which case the animal could construct a spatial representation containing all three elements. For example, presenting LMs A and B together with a food goal could establish a spatial map of all three elements. On the other hand, the same three-element spatial map could be constructed in a piecemeal fashion by joining together two simpler representations, each containing two of the three elements. This process would allow subjects to construct the same three-element representation without experiencing all three elements at the same time. Since visual access to an environment almost always consists of partial views, spatial integration should be an important mechanism for building coherent and useful spatial representations of the environment.

\section{Cue Competition in Spatial Learning}

Higher-order conditioning is one associative process that seems to operate in spatial learning. Cue competition is another associative process whose role in spatial learning has received even greater attention. Cue competition is the reduced behavioral control by a cue, such as a CS, to predict an outcome, such as a US, when other cues are present during cue-outcome pairings. Although cue competition is possible within the taxon system of navigation proposed by O'Keefe 
and Nadel (1978), their theory explicitly denies the possibility of cue-competition in the locale system. Thus, any evidence for cue competition between external landmarks (i.e., not beacons or rigid S-R tendencies) would argue against a non-associative explanation of place learning (see N. Y. Miller \& Shettleworth, 2007, 2008 for a formal associative model of cue competition in spatial learning). Since the publication of O'Keefe and Nadel's book, there have been a slew of studies assessing cue competition in spatial learning. Most of these studies have used adaptations of blocking and overshadowing procedures suited for appetitive and aversive spatial search tasks.

\section{Blocking.}

In a conventional blocking procedure, a CS (A) is first paired with a US in Phase 1, and then a compound of CS A and a second CS (X) is paired with the US in Phase 2. Responding to CS $\mathrm{X}$ on a subsequent test is diminished relative to responding to $\mathrm{CS} X$ in a control group lacking the Phase 1 A-US pairings (Kamin, 1969; Moore \& Schmajuk, 2008). Within the spatial domain, landmarks are used as cues to signal the location of a hidden goal. For example, subjects may learn to navigate to a hidden goal (e.g., the hidden platform in a Morris pool) in the presence of LM A (a spatial cue located outside of the perimeter of the pool), in Phase 1, and receive further training to find the goal in the presence of LMs A and X (a second spatial cue), in Phase 2. A final test with LM X alone can reveal whether spatial control by LM $\mathrm{X}$ was blocked (i.e., weakened) by the presence of the previously trained LM A. O'Keefe and Nadel (1978) propose that any discrepancy between the stored representation of the environment and the environment in which the subject finds itself will automatically trigger exploratory activity, resulting in the assimilation of the new discrepant information into the existing representation. Their model therefore predicts an absence of blocking in the example described above. That is, even though LM A had acquired a predictive relationship to the goal in Phase 1, the addition of the new LM X in Phase 2 would cause the map to be updated with information about LM X. Associative accounts of spatial learning, however, predict that spatial control by LM X should be blocked by LM A.

Rodrigo, Chamizo, McLaren, and Mackintosh (1997) used a Morris pool with landmarks suspended above the edge of the pool. Rats in the blocking group were placed on the platform with LMs A, B, and C. Rats in the control group received trials with LMs A, B, C, and X (Experiment 2) or a separate set of landmarks (e.g., A', B', and C' [Experiment $3])$. In Phase 2, all groups received additional placement trials with LMs A, B, C, and X. Rats were tested with two subsets of the landmarks: $\mathrm{ABC}$ and $\mathrm{ABX}$ on separate trials. On $\mathrm{ABC}$ trials both groups performed equally well, but on
$\mathrm{ABX}$ trials the blocking group showed a significant deficit in locating the quadrant of the hidden platform. Similar blocking effects have been observed in humans trained to navigate a computerized version of the task (Hamilton \& Sutherland, 1999).

To date, just about all experiments on spatial blocking in vertebrates have shown blocking of LM X by a pretrained array of landmarks. Recent experiments by Leising, Stahlman, and Blaisdell (2008) have shown blocking among single landmarks in pigeons in both a touchscreen-procedure and an automated open field (Stahlman \& Blaisdell, 2009, found similar results in a conventional open-field procedure with rats). The automated remote environmental navigation apparatus (ARENA) uses wireless stimulus and response modules that may be arranged in any configuration (Badelt \& Blaisdell, 2008; Leising, Garlick, Parenteau, \& Blaisdell, 2009). Each module detects responses using a capacitive sensor (much like the scroll-wheel of an iPod) and displays stimuli using built-in LEDs. Pecks to a module were reinforced with access to mixed grain retrieved from a central hopper. Subjects were presented with eight linearly arranged modules on the floor of an open field (ARENA; see Figure 4) or discs on a touchscreen-equipped computer screen. In Phase 1, each trial consisted of one module or disc lit with a color designating LM A and subjects were reinforced for pecking at the module or disc (the goal) one unit to the side of LM A. In Phase 2, LMs A and X (a different color) together signaled the location of the hidden goal-with LM A bearing the same spatial relationship with the goal as in Phase 1, and LM X located at the symmetrically opposite side of the goal from LM A. On separate Phase 2 trials, subjects also were trained to locate the hidden goal in the presence of two novel landmarks, B and Y. Figure 10 shows the results from nonreinforced test trials of each landmark alone. LM $\mathrm{X}$ exhibited poorer spatial control than did LMs A and $\mathrm{Y}$ in the touchscreen procedure and poorer than LMs A, B, and $\mathrm{Y}$ in ARENA. These studies demonstrate that blocking can occur between individual landmarks and does not necessarily require an array of pre-trained landmarks (see also Stahlman \& Blaisdell, 2009).

Another associative effect found in spatial learning is unblocking. Unblocking can occur when the information about the outcome signaled by the blocking cue during Phase 1 is changed during Phase 2 (e.g., Blaisdell, Denniston, \& Miller, 1997). Rodrigo, Arall, and Chamizo (2005) replicated the procedure of Rodrigo et al. (1997) using rats in the Morris pool but included an unblocking group. As in Rodrigo et al. (1997), rats in the blocking and unblocking groups were given pretraining with landmarks $\mathrm{A}, \mathrm{B}$, and $\mathrm{C}$ before introducing training trials of $\mathrm{A}, \mathrm{B}, \mathrm{C}$, and $\mathrm{X}$. In the unblocking group, however, the location of the hidden platform relative to the landmarks was changed in Phase 2. This change abolished 


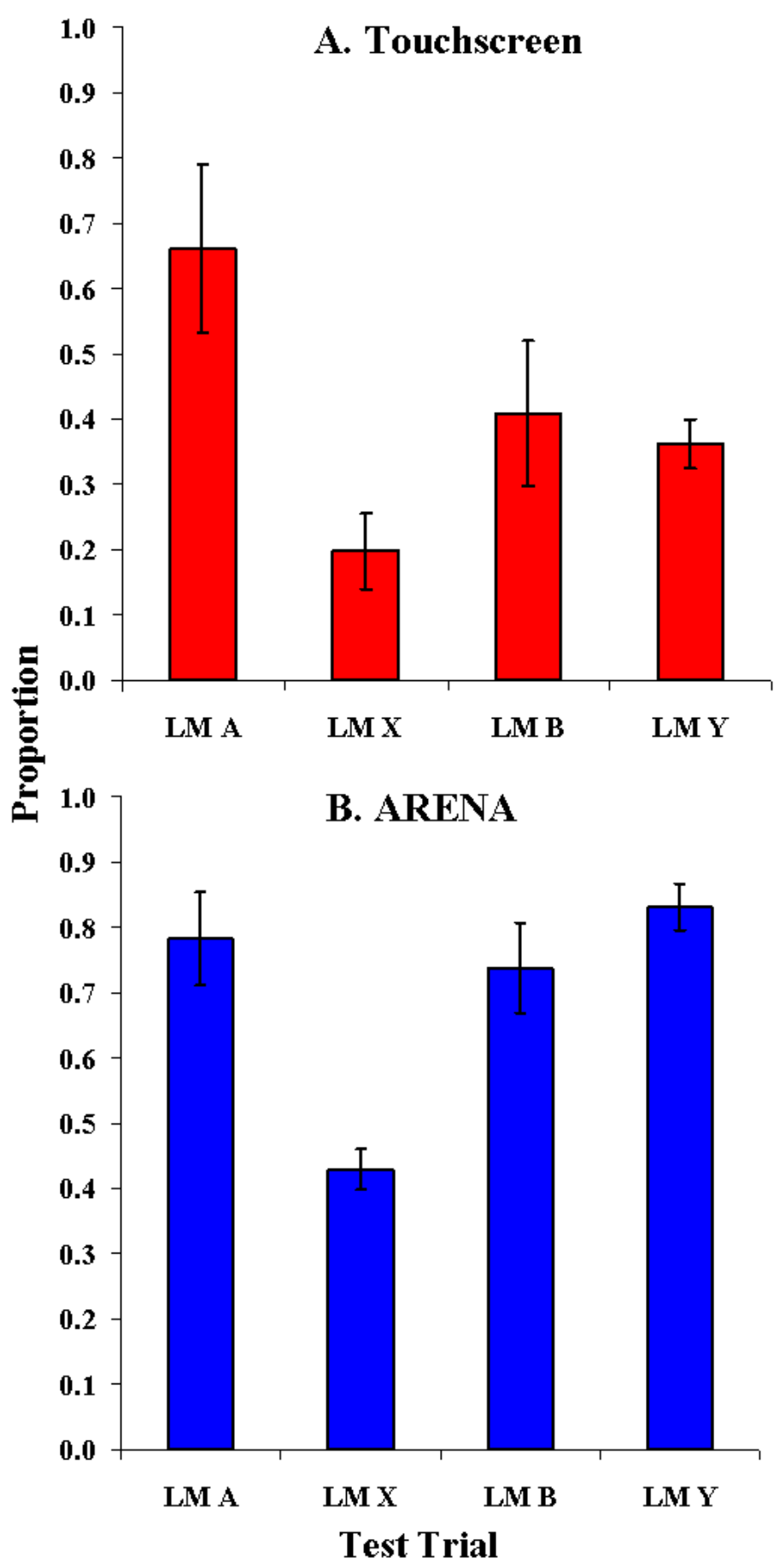

Figure 10. Mean proportion of pecks at the training-goal location on nonreinforced tests of LMs A, X, B, and Y for the touchscreen (top panel) and ARENA (bottom panel) blocking procedures of Leising, Stahlman, and Blaisdell (2008). Error bars represent standard error of the mean.

the blocking effect.

Together, these studies support an associative account of spatial learning. Blocking has also been found in the radialarm maze (Chamizo, Sterio, \& Mackintosh, 1985) and open field (Stahlman \& Blaisdell, 2009; Biegler \& Morris, 1999).

\section{Overshadowing.}

Pavlov (1927, p. 141) reported that when a thermal and tactile CS were repeatedly presented in compound with a US (e.g., acid), the tactile stimulus elicited a response with the same strength as the compound whereas the thermal stimulus elicited no response. In associative terminology, the tactile stimulus is said to overshadow the thermal stimulus relative to a condition in which the thermal stimulus alone was paired with the outcome. Pavlov also found that more intense auditory stimuli overshadow less intense ones in controlling food-related CRs. Recent studies have found a similar overshadowing effect in the spatial domain. In particular, landmarks more proximal to the goal tend to overshadow behavioral control by more distal ones (Chamizo et al., 2006; Cheng, 1992; Spetch, 1995). We review a selection of these studies below (for a more extensive review, see Chamizo, 2002).

Spetch (1995) trained both pigeons and humans in an overshadowing procedure on the touchscreen. Subjects were required to locate a hidden goal signaled by landmarks at varying distances from the goal. On some trials (A), pigeons were presented with landmarks near, moderate, and far from a hidden goal; on the remaining trials (B), they were given a different moderate and far landmark, but no near landmark. The moderate landmark was the same distance from the goal on both A and B trials. Subsequent tests with the moderate landmark alone revealed poorer spatial control in Group A than in Group B, thereby establishing that the degree of spatial control exhibited by a landmark was a function of its relative and not absolute proximity to the hidden goal.

Chamizo, Manteiga, Rodrigo, and Mackintosh (2006) found similar evidence for overshadowing of landmarks in a spatial search task in a Morris pool with rats. Subjects were trained to swim to a platform hidden in a pool of water. On interspersed trials two pairs of landmarks (AB or $\mathrm{BC}$ ) were hung around the perimeter of the pool. In two groups, the relative position of LM B (common to both pairs) to the hidden platform was manipulated. LM B was positioned closer to the hidden platform in the overshadowing group than in the control group. At test, spatial control by a configuration of LMs AC was poor in the overshadowing group relative to the control group thereby showing that control by proximal LM B overshadowed spatial control by LMs A and C.

Leising, Garlick, and Blaisdell (2007) reported overshadowing in both the touchscreen and ARENA preparations. Subjects were given a linear arrangement of eight visually defined circles on a touchscreen-equipped monitor or eight (or six in Experiment 2) ARENA modules. The landmarks were colors displayed on a monitor in the touchscreen or within a module in ARENA. In Experiment 1, subjects re- 


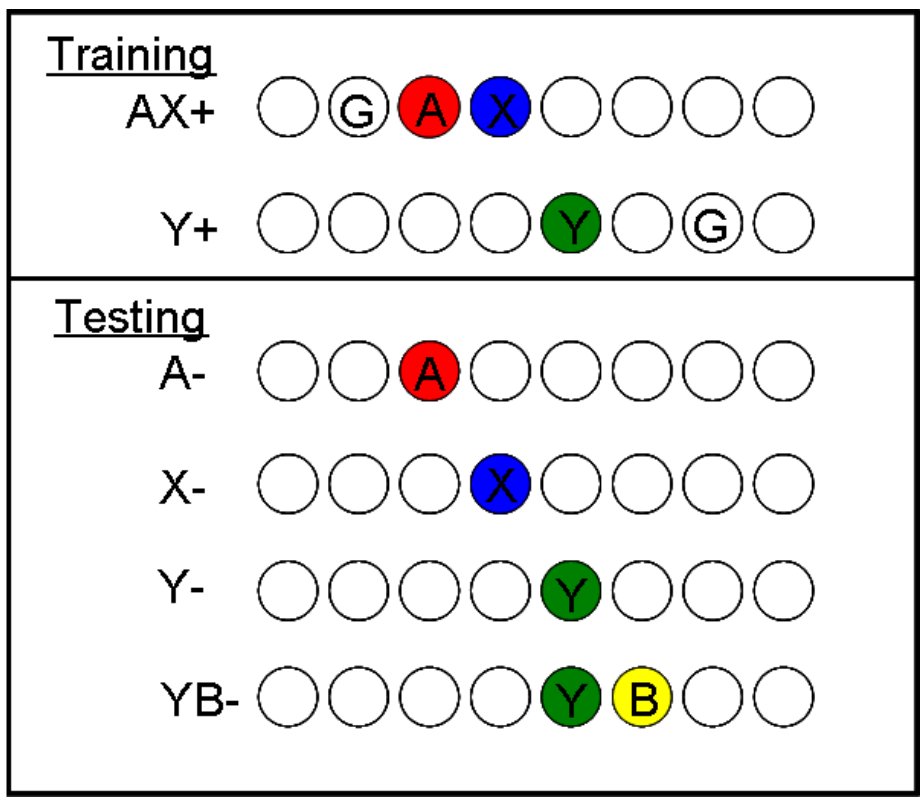

Figure 11. Top panel depicts the overshadowing procedure during training used by Leising, Garlick, and Blaisdell (2007). Bottom panel depicts the nonreinforced test trials. Separate test trials were given for LMs A, X, Y, and YB.

ceived trials of compound LM AX with A closer to the goal than $\mathrm{X}$, and trials of LM Y, with Y bearing the same distance from the goal as did $\mathrm{X}$ (see experimental design in Figure 11). LM X exhibited poorer spatial control than did LM A and LM Y in both preparations. A second experiment using the same procedure demonstrated that this overshadowing effect could not be attributed to generalization decrement, a common alternative account of overshadowing effects (e.g., Pearce, 1987). The generalization decrement account was evaluated by testing LM Y in compound with a novel LM B (i.e., LM BY). On these test trials, LM B was located between LM Y and the goal, thus it was more proximal to the goal than was LM Y. Spatial control by compound LM BY was just as good as by LM Y alone, while we replicated the poorer spatial control by LM X than LM Y (Figure 12). Thus spatial overshadowing in our procedures likely reflects an elementary associative learning phenomenon and not a configural associative strategy or control by the locale spatial-learning system.

According to O'Keefe and Nadel (1978), when an animal encounters a rich array of stimuli that signal the location of a hidden goal, the locale system should construct a cognitive map incorporating all of the predictive stimuli in an all-ornone manner. Overshadowing between landmarks, however, has been found in a variety of vertebrate species, ranging from humans (Chamizo, Aznar-Casanova, \& Artigas, 2002) to pigeons (Cheng, 1989; Leising et al., 2007; Lechelt \& Spetch, 1997; Sánchez-Moreno, Rodrigo, Chamizo, \& Mackintosh, 1999; Spetch, 1995). These findings, as do those from blocking studies discussed above, favor an associative account of

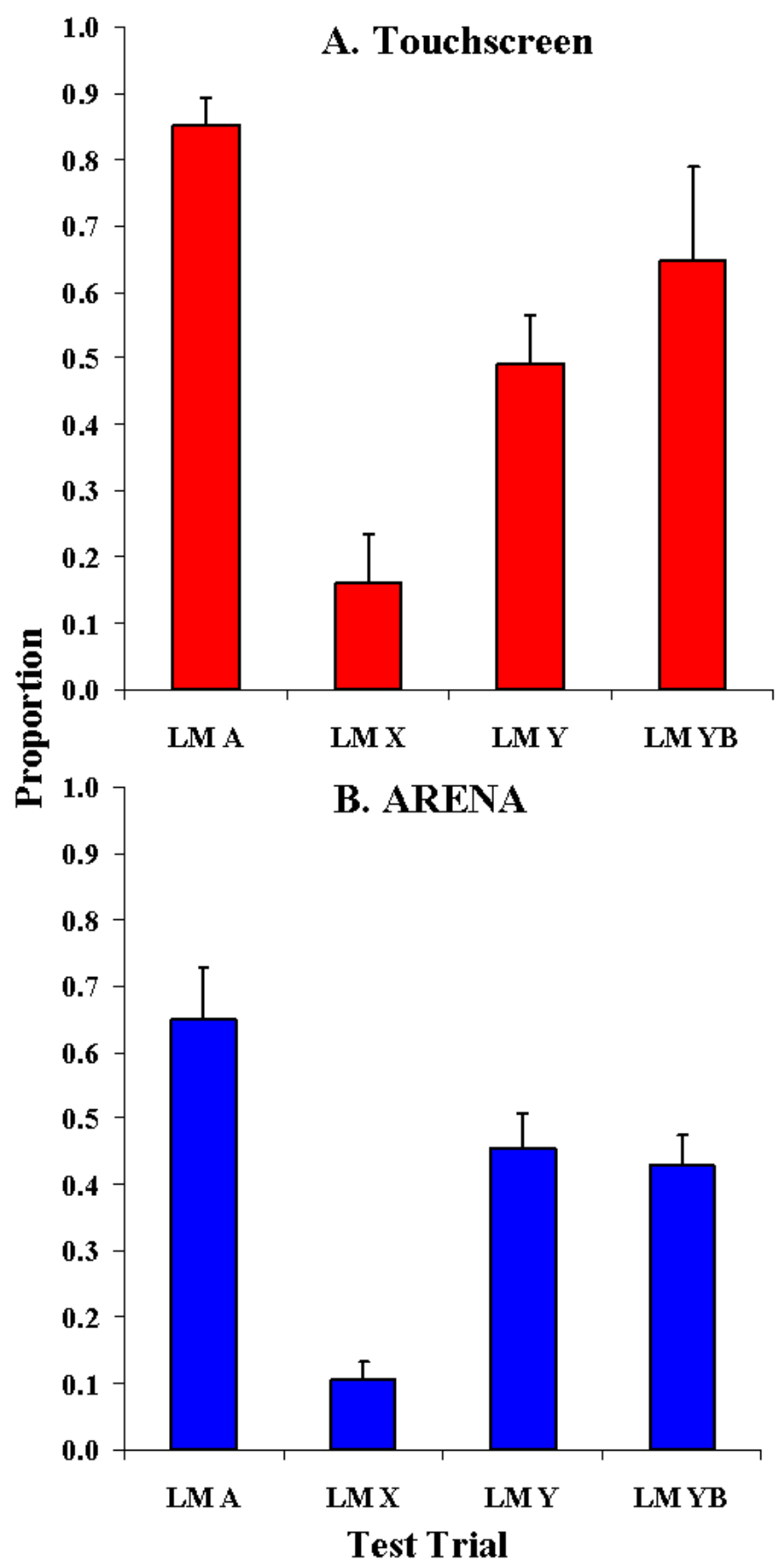

Figure 12. Mean proportion of pecks at the training-goal location on nonreinforced tests of LM A, X, BY, and Y for the touchscreen (top panel) and ARENA (bottom panel) overshadowing procedures of Leising, Garlick, and Blaisdell (2007). Error bars represent standard error of the mean.

spatial learning.

\section{Associative Models of Spatial Learning}

We begin our discussion of the role associative processes play in spatial learning with a few words about aspects of spatial behavior that are likely not the product of associative processes. Euclidean relationships, such as the metric prop- 
erties of distance, direction, and angle should be considered as priors to spatial learning and are more properly dealt with by mechanisms of perception (see Cheng \& Spetch, 1998 for a similar view). Likewise, relational properties, such as middle, between, inside, and outside are likely the products of perceptual or more abstract conceptual processes. Rather, we are interested in describing how associative processes of learning and memory operate on the perceptual and categorical variables of space. This approach is analogous to the approach adopted by proponents of conventional associative models in which it is given that perceptual machinery provides the input for forming stimulus, response, and contextual representations between which associations can be built. Models, such as that of Rescorla and Wagner (1972), are silent about how the subject actually builds a representation of the CS and of the US; but nevertheless presuppose such processes that provide the inputs on which the models can then operate. Likewise, the processes by which metric information is extracted from the environment serves as the input to associative processes that allow a subject to learn that a particular landmark signals a particular goal location, or that multiple landmark-landmark and landmark-goal associations can be integrated into cognitive maps through processes of higher-order conditioning.

Now we address the question of how best to model associative processes of spatial learning. Unfortunately, although many studies have shown that associative processes play a real role in spatial learning, almost no studies have addressed the nature of this learning. For example, although cue-competition phenomena such as blocking and overshadowing have been documented in a variety of spatial learning tasks, there is little evidence to suggest which associative model can best characterize this learning. Overshadowing has been explained in terms of a learning deficit (Rescorla \& Wagner, 1972; Wagner, 1981), an attentional process (Mackintosh, 1975; Pearce \& Hall, 1980), a failure to generalize from a configural stimulus to one of its elements (Pearce, 1987), or as a performance deficit (Denniston, Savastano, \& Miller, 2001). Very little of the accumulated evidence can be marshaled to distinguish between these various theories, but a few studies do provide some clues.

To our knowledge, only a recently presented experiment on overshadowing conducted in our lab directly attempted to dissociate some alternative accounts of landmark overshadowing (Leising et al., 2007). As we described in the section on cue competition, this experiment included a control group that ruled out Pearce's (1987) configural account of landmark overshadowing in our preparation. The pattern of responses by pigeons in experiments by Spetch et al. (1996) and Spetch et al. (1997) also provide evidence favoring elemental models of spatial learning (though their studies weren't specifically testing overshadowing). In their studies, pigeons were trained to find food hidden in the center of an array of four visually distinct landmarks. At test, the array was expanded so that the landmarks retained their square formation (with each landmark at the corner of an imaginary square boundary) but with increased distances between the landmarks and the goal. Individual pigeons showed preference for searching at the same distance and direction from one or two of the landmarks of the array, thereby showing evidence of encoding the absolute distance and direction separately for some of the landmarks - an elemental strategy.

Higher-order conditioning results also pose a difficulty for configural models (though pattern-completion models, e.g., Rudy and O'Reilly, 2001, might be extended to account for them). Several existing elemental models of spatial learning are well supported, such as Vector Sum model (Cheng, 1986; Collett et al., 1986) and the N. Y. Miller \& Shettleworth (2007, 2008) model. On the other hand, elemental models of associative learning have difficulties providing accounts of both conditioned inhibition and higher-order conditioning. That is, existing theories tend to model positive mediation, such as Holland $(1981 ; 1990)$ and Hall (1996), or negative mediation, such as Mackintosh (1975); Pearce (1987), Pearce \& Hall (1980); Rescorla \& Wagner (1972); Wagner (1981). Other models that fall between a pure associative model and a computational model, such as the comparator hypothesis (Miller \& Matzel, 1988; Denniston et al., 2001; Stout \& Miller, 2007), or fully computational models, such as Gallistel and Gibbon (2000) may also be usefully applied to spatial learning. To date, there have been few strong tests to differentiate how well these models extend to spatial learning. N. Y. Miller and Shettleworth provide the first attempt to adapt a conventional associative learning model (an operant extension of the Rescorla and Wagner rule) to provide a general account for learning in various spatial domains, such as geometric cues and discrete landmarks. This model seems more concerned, however, with reconciling the discrepancy between studies of geometry learning and landmark or cuebased learning rather than assessing various general learning mechanisms. Perhaps the only extant model that can account for both positive and negative mediation is Stout and Miller's SOCR model which is a formal implementation of the extended comparator hypothesis (Blaisdell, Bristol, Gunther, \& Miller, 1998; Denniston et al., 2001).

A second dimension on which to dichotomize associative models involves the learning-performance distinction. While most models of Pavlovian conditioning phenomena, for example, model processes that operate during the acquisition of associative relationships, such as between a CS and a US, some candidate models attempt to account for these phenomena using processes that operate at the time of performance. One classic performance focused model is the compara- 
tor hypothesis (Miller \& Matzel, 1988). In the framework of the comparator hypothesis, associations are formed and strengthened between all continuous stimuli (e.g., all stimuli present on a trial). Performance to any particular predictive stimulus such as a CS at test involves a comparison between the association between the test CS and the US and the associations of each of its comparator stimuli and the US. Comparator stimuli involve all other stimuli that were present during training of the CS, and include things like other CSs and the context. The model accounts for cue-competition phenomena like overshadowing not as a function of differential learning about the overshadowed CS (X) compared to the same CS in a control group for which an overshadowing CS (A) was not present, but (loosely) in terms of the relative strength of the association between the overshadowed CS X and the US compared to that of the overshadowing CS A and the US. Evidence for this account comes from posttraining manipulations that increase the relative strength of the X-US association or decrease in the relative strength of the competing A-US association. Posttraining extinction of CS A for example, attenuates the overshadowing effect, producing an increase in conditioned responding to CS X (Kaufman \& Bolles, 1981; Kasprow, Cacheiro, Balaz, \& Miller, 1982). Similarly, Blaisdell, Gunther, and Miller (1998) report an effect of extinction of a blocking CS on recovery of responding to a blocked CS. These results had been taken as strong evidence for comparator models of cue competition over acquisition-focused models like that of Rescorla and Wagner (1972). Recently, however, acquisition focused models have been revised or modified to enable them to account for these "retrospective revaluation" effects. Van Hamme and Wasserman (1994) described a revision of the RescorlaWagner model and Dickinson and Burke (1996) have described a revision of Wagner's SOP (1981) model; both can account for extinction-mediated recovery from overshadowing. These revised models have motivated an extension of the comparator hypothesis (Blaisdell et al. 1998; Denniston et al., 2001; Stout \& Miller, 2007) that accounts for certain recent phenomena that the revised acquisition-focused models fail to explain (see review by Blaisdell, 2003). Similar experiments as those described above could be conducted in landmark learning tasks to identify the models that best account for cue-competition phenomena in spatial learning. For example, would extinction of a blocking landmark result in increased spatial control by a blocked landmark? Many other questions, such as the roles of attention, context, and cognition to name just a few, need to be addressed to explore the appropriate associative models for spatial learning. Another fruitful approach may be to adopt Cheng and Spetch's (1998) framework of treating goal-seeking navigation as a servomechanism analogous to a homeostatic process. Pavlovian processes also have a long history of treatment as a homeostatic process, and thus there is a common link to hitch these two frameworks together under this common theme. Nevertheless, the current stage of investigation of the appropriate models and framework of spatial learning is still in its infancy but with many exciting possibilities to explore.

\section{Challenges for a General Mechanism of Learning}

Though most of the evidence from cue-competition procedures in the spatial domain has supported an important role for an associative mechanism in landmark-based search tasks, a few studies demonstrate effects difficult to explain solely in terms of an association mechanism. O'Keefe and Conway (1978) reported that hippocampal place cells in freely moving rats fired when the rat was in specific regions of a maze (defined by four landmarks placed around the maze). Interestingly, the removal of two of the landmarks was not sufficient to prevent the place cells defining each region from firing. Thus, subjects must have encoded a configuration of landmarks such that a subset of the configuration was sufficient to activate the place representation. Similarly, Rodrigo et al. (1997) trained rats to locate a hidden platform in a Morris pool in the presence of four landmarks positioned around the perimeter of the pool. In their first experiment, they found that subjects could effectively use a subset of two or three landmarks to find the hidden goal. Evidently, despite only needing to learn about two landmarks to locate the hidden goal, rats learned the goal relative to every landmark. In a subsequent experiment, however, rats in a blocking group were pre-trained to locate a hidden platform in the presence of landmarks $\mathrm{A}, \mathrm{B}$, and $\mathrm{C}$ before adding the fourth landmark (LM X). In a control group, rats were trained on A, B, C, and $\mathrm{X}$ without pre-training. At test, spatial control by LMs ABX in the blocking group was impaired relative to LMs ABC in the blocking group and LMs ABX in the control group.

Encoding every available landmark makes functional sense when the representation contains errors - which is just about always. By using multiple sources, errors can be reduced. But as we have seen, there are many situations in which spatial control by individual landmarks has been compromised and thus given less weight by the subject. This can occur when a landmark is redundant (blocking) or when there are other landmarks that are more salient or closer to the goal (overshadowing). Different weighting of landmarks could result in differential learning about them (Cheng, Shettleworth, Huttenlocher, \& Rieser, 2007; Kamil \& Cheng, 2001). Alternatively, the different weighting of landmarks might reflect a performance rather than learning variable. A performance account would predict that all landmarks are learned about, consistent with other approaches to cognitive mapping (e.g., O'Keefe \& Nadel, 1978). Evidence for a performance account of differential landmark weighting would come from posttraining manipulations that uncover the latent information conveyed by the landmark that initially received 
a lower relative weight. This approach parallels that taken for comparator theories of associative learning phenomena (e.g., Gibbon \& Balsam, 1981; Denniston et al., 2001) which provide an alternative account of cue-competition effects in Pavlovian conditioning than do acquisition-deficit models (Mackintosh, 1975; Pearce \& Hall, 1980; Rescorla \& Wagner, 1972; Wagner, 1981). These various accounts of landmark learning have yet to be tested.

\section{Conclusion}

We reviewed evidence that associative processes play an important role in spatial learning. The earlier history of spatial learning was concerned with how to distinguish learning that was characterized as being either habitual or goal directed. More recent research has focused on the commonalities between the two types of learning and the generality of an associative mechanism of spatial learning. Support for a general role of associative learning comes from experiments investigating the learning about a single landmark, integration of spatial maps, and cue competition effects such as blocking and overshadowing in the spatial domain (see also Blaisdell, 2009).

\section{References}

Arcediano, F., Escobar, E., \& Miller, R. R. (2003). Temporal integration and temporal backward associations in human and nonhuman subjects. Learning \& Behavior, 31, 242256.

Badelt, S. W., \& Blaisdell, A. P. (2008). Capacitive sensors for detecting proximity and response. Behavior Research Methods, 40, 613-621. doi:10.3758/BRM.40.2.613

Battig, K., Driscoll, P., Schlatter, J., \& Uster, H. J. (1976). Effects of nicotine on the exploratory locomotion patterns of female Roman high- and low-avoidance rats. Pharmacology, Biochemistry and Behavior, 4, 435-439.

doi:10.1016/0091-3057(76)90060-5

Benhamou, S. (1996). No evidence for cognitive mapping in rats. Animal Behaviour, 52, 201-212.

doi:10.1006/anbe.1996.0165

Bennett, A. T. (1996). Do animals have cognitive maps? Journal of Experimental Biology, 199, 219-224.

Biegler, R., \& Morris, R. G. (1999). Blocking in the spatial domain with arrays of discrete landmarks. Journal of Experimental Psychology: Animal Behavior Processes, 25, 334-351. doi:10.1037/0097-7403.25.3.334

Blaisdell, A. P. (2003). The S-R information stream: Where's the filter? Integrative Physiological \& Behavioral Science, 38, 146-165. doi:10.1007/BF02688832

Blaisdell, A. P. (2009). The role of associative processes in spatial, temporal, and causal cognition. In Watanabe, S., Blaisdell, A. P., Huber, L., \& Young, A. (Eds.), Rational Animals, Irrational Humans (pp. 153-172). Keio Univer- sity Press; Tokyo, Japan.

Blaisdell, A. P., Bristol, A. S., Gunther, L. M., \& Miller, R. R. (1998). Overshadowing and latent inhibition counteract each other: Support for the comparator hypothesis. Journal of Experimental Psychology: Animal Behavior Processes, 24, 335-351. doi:10.1037/0097-7403.24.3.335

Blaisdell, A. P., \& Cook, R. G. (2005). Integration of spatial maps in pigeons. Animal Cognition, 8, 7-16. doi:10.1007/s10071-004-0223-1

Blaisdell, A. P., Denniston, J. C., \& Miller, R. R. (1997). Unblocking with qualitative change of unconditioned stimulus. Learning and Motivation, 28, 268-279.

doi:10.1006/lmot.1996.0961

Blaisdell, A. P., Gunther, L. M., \& Miller, R. R. (1999). Recovery from blocking achieved by extinguishing the blocking stimulus. Animal Learning \& Behavior, 27, 6376.

Blodgett, H. C., \& McCutchan, K. (1948). Relative strength of place and response learning in the T-maze. Journal of Comparative \& Physiological Psychology, 41, 17-24. doi:10.1037/h0061366

Bond, A. B., Cook, R. G., \& Lamb, M. R. (1981). Spatial memory and performance of rats and pigeons in the radialarm maze. Animal Learning \& Behavior, 9, 575-580.

Burgess, N. (2006). Spatial memory: how egocentric and allocentric combine. Trends in Cognitive Sciences, 10, 551557. doi:10.1016/j.tics.2006.10.005

Burgess, N. (2008). Spatial cognition and the brain. New York Academy of Sciences, 1124, 77-97.

Carr, H., \& Watson, J. B (1908). Orientation in the white rat. Journal of Comparative Neurology and Psychology, 18, 27-44. doi:10.1002/cne.920180103

Calhoun, J. (1962). The ecology and sociology of the Norway rat. Bethesda, MD: U.S. Department of Health, Education and Welfare.

Chamizo, V. D. (2002). Spatial learning: Conditions and basic effects. Psicologica, 23, 33-57.

Chamizo, V.D. (2003). Acquisition of knowledge about spatial location: Assessing the generality of the mechanism of learning. The Quarterly Journal of Experimental Psychology, 56B, 102-113.

Chamizo, V. D., Aznar-Casanova, J. A., \& Artigas, A. A. (2002). Human overshadowing in a virtual pool: Simple guidance is a good competitor against locale learning. Learning and Motivation, 34, 262-281. doi:10.1016/S0023-9690(03)00020-1

Chamizo, V. D., Manteiga, R. D., Rodrigo, T. \& Mackintosh, N. J. (2006). Competition between landmarks in spatial learning: The role of proximity to the goal. Behavioural Processes, 71, 59-65.

Chamizo, V. D., Rodrigo, T., \& Mackintosh, N. J. (2006). Spatial integration in rats. Learning \& Behavior, 34, 348354. 
Chamizo, V. D., Sterio, D., \& Mackintosh, N. J. (1985). Blocking and overshadowing between intra-maze and extra-maze cues: A test of the independence of locale and guidance learning. Quarterly Journal of Experimental Psychology, 37B, 235-253.

Cheng, K. (1986). A purely geometric module in the rat's spatial representation. Cognition, 23, 149-178. doi:10.1016/0010-0277(86)90041-7

Cheng, K. (1988). Some psychophysics of the pigeon's landmark use. Journal of Comparative Physiology A, 162, 815-826. doi:10.1007/BF00610970

Cheng, K. (1989). The vector sum model of pigeon landmark use. Journal of Experimental Psychology: Animal Behavior Processes, 15, 366-375. doi:10.1037/0097-7403.15.4.366

Cheng, K. (1990). More psychophysics of the pigeon's use of landmarks. Journal of Comparative Physiology. A: Sensory, Neural, and Behavioral Physiology, 166, 857-863. doi:10.1007/BF00187333

Cheng, K. (1992). Three psychophysical principles in the processing of spatial and temporal information. In Honig, W. K. \& Fetterman, J. G. (Eds.), Cognitive aspects of stimulus control (pp. 69-88). NJ, England: Erlbaum.

Cheng, K. (1994). The determination of direction in landmark-based spatial search in pigeons: a further test of the vector sum model. Animal Learning \& Behavior, 22, 291-301.

Cheng, K. (2008). Whither geometry? Troubles of the geometric module. Trends in Cognitive Science, 12, 355-361. doi:10.1016/j.tics.2008.06.004

Cheng, K., Shettleworth, S. J., Huttenlocher, J., \& Rieser, J. J. (2007). Bayesian integration of spatial information. Psychological Bulletin, 133, 625-637. doi:10.1037/0033-2909.133.4.625

Cheng, K., \& Spetch, M. L., (1995). Stimulus control in the use of landmarks by pigeons in a touch-screen task. Journal of Experimental Analysis of Behavior, 63, 187-201. doi:10.1901/jeab.1995.63-187

Cheng, K., \& Spetch, M.L., (1998). Mechanisms of landmark use in mammals and birds. In: Healy, S. (Ed.), Spatial representation in animals. Oxford University Press, Oxford, New York, 1-17.

Cheng, K., Spetch, M. L., Kelly, D. M., \& Bingman, V. P. (2006). Small-scale spatial cognition in pigeons. Behavioural Processes, 72, 115-127.

doi:10.1016/j.beproc.2005.11.018

Collett, T. S., Cartwright, B. A., \& Smith, B. A. (1986). Landmark learning and visuo-spatial memories in gerbils. Journal of Comparative Physiology, 158A, 835-851. doi:10.1007/BF01324825

Dashiell, J, F. (1930). Direction orientation in maze running by the white rat. Comparative Psychology Monographs, 7, No. 32.
Dember, W. N., \& Fowler, H. (1958). Spontaneous alternation behavior. Psychological Bulletin, 55, 412-428. doi: $10.1037 / \mathrm{h} 0045446$

Dennis, W. (1932). Multiple visual discrimination in the block elevated maze. Journal of Comparative Psychology, 13, 391-396. doi:10.1037/h0073485

Denniston, J. C., Savastano, H. I., \& Miller, R. R. (2001). The extended comparator hypothesis: Learning by contiguity, responding by relative strength. In R. R. Mowrer \& S. B. Klein (Eds.), Handbook of contemporary learning theories (pp. 65-117). Hillsdale, NJ: Erlbaum.

Dickinson, A. \& Burke, J. (1996). Within-compound associations mediate the retrospective revaluation of causality judgments. Quarterly Journal of Experimental Psychology, 49B, 60-80.

Douglas, R. J. (1966). Cues for spontaneous alternation. Journal of Comparative and Physiological Psychology, 62, 171-183. doi: $10.1037 / \mathrm{h} 0023668$

Galanter, E. H. (1955). Place and response learning: learning to alternate. Journal of Comparative and Physiological Psychology, 48, 17-18. doi:10.1037/h0040760

Gallistel, C.R., \& Cramer, A. E. (1996). Computations on metric maps in mammals: getting oriented and choosing a multi-destination route. Journal of Experimental Biology, 199, 211-217.

Gallistel, C. R., \& Gibbon, J. (2000). Time, rate, and conditioning. Psychological Review, 107, 219-275. doi:10.1037/0033-295X.107.2.289

Gibbon, J., \& Balsam, P. D. (1981). Spreading association in time. In C. M. Locurto, H. S. Terrace, \& J. Gibbon (Eds.), Autoshaping and conditioning theory (pp. 219-253). New York: Academic Press.

Gibson, B. M., \& Kamil, A. C. (2001). Tests for cognitive mapping in Clark's Nutcrackers (Nucifraga columbiana). Journal of Comparative Psychology, 115, 403-417. doi:10.1037/0735-7036.115.4.403

Gingerelli, J. A. (1929). Preliminary experiments on the causal factors in animal learning. II. Journal of Comparative Psychology, 9, 245-274. doi: $10.1037 / \mathrm{h} 0073956$

Hall, G. (1996). Learning about associatively activated stimulus representations: Implications for acquired equivalence and perceptual learning. Animal Learning \& Behavior, 24, 233-255.

Hamilton, D. A. \& Sutherland, R. J. (1999). Blocking in human place learning: evidence from virtual navigation. Psychobiology, 27, 453-61.

Hicks, L. H. (1964). Effects of overtraining on acquisition and reversal of place and response learning. Psychological Reports, 15, 459-462.

Holland, P. C. (1981). Acquisition of representation-mediated conditioned food aversions. Learning and Motivation, 12, 1-18. doi:10.1016/0023-9690(81)90022-9 
Holland, P. C., (1990). Event representation in Pavlovian conditioning: Image and action. Cognition, 37, 105-131. doi:10.1016/0010-0277(90)90020-K

Hull, C.L., 1943. Principles of Behaviour. Appleton-Century-Crofts, New York.

Jacobs, L. F., \& Schenk, F. (2003). Unpacking the cognitive map: the parallel map theory of hippocampal function. Psychological Review, 110, 285-315. doi:10.1037/0033-295X.110.2.285

Kamil, A.C., \& Cheng, K. (2001). Way-finding and landmarks: the multiple bearings hypothesis. Journal of Experimental Biology, 204, 103-113.

Kamin, L. J. (1969). Predictability, surprise, attention, and conditioning. In Cambell, B.A. \& Church, R.M. (Eds.), Punishment and Aversive Behavior (pp. 279-296). New York, NY: Appleton-Century-Crofts.

Kasprow, W. J., Cacheiro, H., Balaz, M. A., \& Miller, R. R. (1982). Reminder-induced recovery of associations to an overshadowed stimulus. Learning and Motivation, 13, 155-166. doi:10.1016/0023-9690(82)90018-2

Kaufman, M. A., \& Bolles, R. C. (1981). A nonassociative aspect of overshadowing. Bulletin of the Psychonomic Society, 18, 318-320.

Lechelt, D. P. \& Spetch, M. L. (1997). Pigeons' use of landmarks for spatial search in a laboratory arena and in digitized images of the arena. Learning and Motivation, 28, 424-445. doi:10.1006/lmot.1997.0975

Leising, K. J., Garlick, D., \& Blaisdell, A. P. (2007). Development of an automated open field for examining cognitive processes in pigeons. Paper presented at the 14th annual meeting of the Comparative Cognition Society, Melbourne, FL.

Leising, K. J., Garlick, D., Parenteau, M., \& Blaisdell, A. P. (2009). Behavioral research in pigeons in ARENA: An automated remote environmental navigation apparatus. Behavioural Processes, 85, 105-113. doi:10.1016/j.beproc.2009.02.008

Leising, K. J., Sawa, K., \& Blaisdell, A. P. (2007). Temporal integration in Pavlovian appetitive conditioning in rats. Learning \& Behavior, 35, 11-18.

Leising, K. J., Stahlman, W. D., \& Blaisdell, A. P. (2008) Cue-competition effects in spatial control by landmarks in rats and pigeons. Paper presented at the 15th annual meeting of the Comparative Cognition Society, Melbourne, FL.

Mackintosh, N. J. (1975). A theory of attention: Variations in the associability of stimuli with reinforcement. Psychological Review, 82, 276. doi:10.1037/h0076778

Mackintosh, N. J. (2002). Do not ask whether they have a cognitive map, but how they find their way about. Psicologica, 23, 165-185.

Miller, R. R., \& Matzel, L. D. (1988). The comparator hypothesis: A response rule for the expression of associa- tions. In G. H. Bower (Ed.), The psychology of learning and motivation (Vol. 22, pp. 51-92). San Diego, CA: Academic Press.

Miller, N. Y., \& Shettleworth, S. J. (2007). Learning about environmental geometry: An associative model. Journal of Experimental Psychology: Animal Behavior Processes, 33, 191-212. doi:10.1037/0097-7403.33.3.191

Miller, N. Y., \& Shettleworth, S. J. (2008). An associative model of geometry learning: A modified choice rule. Journal of Experimental Psychology: Animal Behavior Processes, 34, 419-422. doi:10.1037/0097-7403.34.3.419

Molet, M. \& Miller, R. R. (2008). Integration of cognitive maps in humans. Paper presented at the 15th Annual International Conference on Comparative Cognition, Melbourne, FL.

Moore, J. \& Schmajuk, N. A. (2008). Kamin blocking. Scholarpedia, 3: 3542.

Morris, R. G. (1981). Spatial localization does not require the presence of local cues. Learning and Motivation, 12, 239-60. doi:10.1016/0023-9690(81)90020-5

Morris, R. G. M., Hagan, J. J. \& Rawlins, J. N. P. (1986). Allocentric spatial learning by hippocampectomized rats: A further test of the 'spatial mapping' and 'working memory' theories of hippocampal function. Quarterly Journal of Experimental Psychology, 38, 365-395.

Munn, N. L. (1950). Handbook of psychological research on the rat: An introduction to animal psychology. Boston: Houghton Mifflin.

O'Keefe, J., \& Conway, D. H. (1978). Hippocampus place units in the freely moving rat: Why they fire where they fire. Experimental Brain Research, 31, 573-590.

O'Keefe, J., \& Nadel, L. (1978). The hippocampus as a cognitive map. Oxford: Oxford University Press.

Olson, D. J., \& Maki, W. S. (1983). Characteristics of spatial memory in pigeons. Journal of Experimental Psychology: Animal Behavior Processes, 9, 266-280. doi:10.1037/0097-7403.9.3.266

Olton, D. S. (1979). Mazes, maps, and memory. American Psychologist, 34, 583-596. doi:10.1037/0003-066X.34.7.583

Olton, D. S., \& Samuelson, R. J. (1976). Remembrance of places passed: Spatial memory in rats. Journal of Experimental Psychology: Animal Behavior Processes, 2, 97116. doi:10.1037/0097-7403.2.2.97

Packard, M. G., \& McGaugh, J. L. (1996). Inactivation of hippocampus or caudate nucleus with lidocaine differentially affects expression of place and response learning. Neurobiology of Learning \& Memory, 65, 66-72. doi:10.1006/nlme.1996.0007

Pavlov, I. P. (1927). Conditioned reflexes. London: Oxford University Press.

Pearce, J. M. (1987). A model for stimulus generalization in Pavlovian conditioning. Psychological Review, 94, 61-73. 
doi:10.1037/0033-295X.94.1.61

Pearce, J. M. \& Hall, G. (1980). A model for Pavlovian learning: Variations in the effectiveness of conditioned but not of unconditioned stimuli. Psychological Review, 87, 532552. doi:10.1037/0033-295X.87.6.532

Poucet, B. (1993). Spatial cognitive maps in animals: New hypotheses on their structure and neural mechanisms. Psychological Review, 100, 163-82.

doi:10.1037/0033-295X.100.2.163

Rescorla, R. A., \& Wagner, A. R. (1972). A theory of Pavlovian conditioning: Variations in the effectiveness of reinforcement and nonreinforcement. In A. H. Black \& W. F. Prokasy (Eds.), Classical conditioning II: Current research and theory (pp. 64-99). New York: Appleton-Century-Crofts.

Restle, F. (1957). Discrimination of cues in mazes: A resolution of the "place-vs.-response" question. Psychological Review, 64, 217-228. doi:10.1037/h0040678

Roberts, W. A., \& Van Veldhuizen, N. (1985). Spatial memory in pigeons on the radial maze. Journal of Experimental Psychology: Animal Behavior Processes, 11, 241-260. doi:10.1037/0097-7403.11.2.241

Ritchie, B. F., Aeschliman, B., \& Pierce, P. (1950). Studies in spatial learning: VIII. Place performance and the acquisition of place dispositions. Journal of Comparative and Physiological Psychology, 43, 73-85.

doi:10.1037/h0055224

Rodrigo, T., Arall, M. \& Chamizo, V. D. (2005). Blocking and unblocking in a navigation task. Psicológica, 26, 229241.

Rodrigo, T., Chamizo, V. D., McLaren, I. P. L., \& Mackintosh, N. J. (1997). Blocking in the spatial domain. Journal of Experimental Psychology: Animal Behavior Processes, 23, 110-118. doi:10.1037/0097-7403.23.1.110

Rudy, J. W., \& O'Reilly, R. C. (2001). Conjunctive representations, the hippocampus, and contextual fear conditioning. Cognitive, Affective, \& Behavioral Neuroscience, $\mathbf{1}$, 66-82. doi:10.3758/CABN.1.1.66

Sánchez-Moreno, J., Rodrigo, T., Chamizo, V. D., \& Mackintosh, N. J. (1999). Overshadowing in the spatial domain. Animal Learning \& Behaviour, 27, 391.

Savastano, H. I., \& Miller, R. R. (1998). Time as content in Pavlovian conditioning. Behavioural Processes, 44, 147162. doi:10.1016/S0376-6357(98)00046-1

Sawa, K., Leising, K. J., \& Blaisdell, A. P. (2005). Sensory preconditioning in spatial learning using a touch screen task in pigeons. Journal of Experimental Psychology: Animal Behavior Processes, 31, 368-375.

doi:10.1037/0097-7403.31.3.368

Scharlock, D. P. (1955). The role of extramaze cues in place and response learning. Journal of Experimental Psychology, 50, 249-254. doi:10.1037/h0043052

Sherry, D. F., \& Healy, S. (1998). Neural mechanisms of spatial representation. In: Healy S, editor. Spatial representations in animals. Oxford: Oxford University Press, pp 133-157.

Shettleworth, S. J. (1998). Cognition, evolution, and behavior. Oxford: Oxford University Press.

Small, W. S. (1901). Experimental study of the mental processes of the rat. II. American Journal of Psychology, 12, 206-239.

Spetch, M. L. (1990). Further studies of pigeons' spatial working memory in the open-field task. Animal Learning \& Behavior, 18, 332-340.

Spetch, M. L. (1995). Overshadowing in landmark learning: Touch-screen studies with pigeons and humans. Journal of Experimental Psychology: Animal Behavior Processes, 21, 166-181. doi:10.1037/0097-7403.21.2.166

Spetch, M. L., Cheng, K., \& MacDonald, S. E. (1996). Learning the configuration of a landmark array: I. Touch-screen studies with pigeons and humans. Journal of Comparative Psychology, 110, 55-68.

doi:10.1037/0735-7036.110.1.55

Spetch, M. L., Cheng, K., MacDonald, S. E., Linkenhoker, B. A., Kelly, K. M., \& Doekson, S. R. (1997). Use of landmark configuration in pigeons and humans: II. Generality across search tasks. Journal of Comparative Psychology, 111, 14-24. doi:10.1037/0735-7036.111.1.14

Spetch, M. L., Cheng, K., \& Mondloch, M. V. (1992). Landmark use by pigeons in a touch-screen spatial search task. Animal Learning \& Behavior, 20, 281-292.

Spetch, M. L., \& Edwards, C. A. (1986). Spatial memory in pigeons (Columba livia) in an open-field feeding environment. Journal of Comparative Psychology, 100, 266-278. doi:10.1037/0735-7036.100.3.266

Spetch, M. L., \& Honig, W. K. (1988). Characteristics of pigeons' spatial working memory in an open-field task. Animal Learning \& Behavior, 16, 123-131.

Spetch, M. L., \& Mondloch, M. V., (1993). Control of pigeons' spatial search by graphic landmarks in a touchscreen task. Journal of Experimental Psychology: Animal Behavior Processes, 19, 353-372. doi:10.1037/0097-7403.19.4.353

Spetch, M. L. \& Wilkie, D. M. (1994). Pigeons' use of landmarks presented in digitized images. Learning and Motivation, 25, 245-275. doi:10.1006/lmot.1994.1014

Stahlman, W. D. \& Blaisdell, A. P. (2009). Blocking of spatial control by landmarks in rats. Behavioural Processes, 85, 114-118. doi:10.1016/j.beproc.2009.02.007

Stoltz, S. B., \& Lott, D. F. (1964). Establishment in rats of a persistent response producing a net loss of reinforcement. Journal of Comparative and Physiological Psychology, 57, 147-149. doi:10.1037/h0042991

Stout, S. C., \& Miller, R. R. (2007). Sometimes-competing retrieval (SOCR): A formalization of the comparator hypothesis. Psychological Review, 114, 759-783. 
doi:10.1037/0033-295X.114.3.759

Sturz, B. R., Bodily, K. D., \& Katz, J. S. (2006). Evidence against integration of spatial maps in humans. Animal Cognition, 9, 207-217. doi:10.1007/s10071-006-0022-y

Suzuki, S., Augerinos, G., \& Black, A. H. (1980). Stimulus control of spatial behavior in the eight-arm maze in rats. Learning and Motivation, 11, 1-18. doi:10.1016/0023-9690(80)90018-1

Tolman, E. C. (1948). Cognitive maps in rats and men. Psychological Review, 55, 189-208. doi:10.1037/h0061626

Tolman, E. C., Ritchie, B. F., \& Kalish, D. (1946a). Studies in spatial learning. I. Orientation and the short-cut. Journal of Experimental Psychology, 36, 13-24. doi:10.1037/h0053944

Tolman, E. C., Ritchie, B. F., \& Kalish, D. (1946b) Studies in spatial learning: II. Place learning versus response learning. Journal of Experimental Psychology, 36, 221-229. doi:10.1037/h0060262

Tremblay, J., \& Cohen, J. (2005). Spatial configuration and list learning of proximally cued arms by rats in the enclosed four-arm radial maze. Learning \& Behavior, 33, 78-89.

Uster, H. J., Battig, K., \& Nageli, H. H. (1976). Effects of maze geometry and experience on exploratory behavior in the rat. Animal Learning \& Behavior, 4, 84-88.

Van Hamme, L. J., \& Wasserman, E. A. (1994). Cue competition in causality judgments: The role of nonrepresentation of compound stimulus elements. Learning and Motivation, 25, 127-151. doi:10.1006/lmot.1994.1008

Wagner, A. R. (1981). SOP: A model of automatic memory processing in animal behavior. In N.E. Spear \& R.R. Miller (Eds.), Information processing in animals: Memory mechanisms (pp.5-47). Hillsdale, NJ: Erlbaum.

Watson, J. B. (1907). Kinaesthetic and organic sensations: Their role in the reactions of the white rat to the maze. Psychological Review Monograph Supplement, No. 33.

Woodworth, R.S. (1938). Experimental psychology, New York: Holt. 